\title{
ON UPSCALING CERTAIN FLOWS IN DEFORMABLE POROUS MEDIA*
}

\author{
OLEG ILIEV ${ }^{\dagger}$, ANDRO MIKELIĆ $\ddagger$, AND PETER POPOV
}

\begin{abstract}
We consider certain computational aspects of upscaling fluid flows through deformable porous media. We start with pore level models and discuss upscaled (homogenized) equations and respective cell problems. Analytical solution of a cell problem in certain geometry, as well as an accurate numerical procedure for the general case, are presented. A microscale (pore level) fluidstructure interaction (FSI) problem is formulated in terms of incompressible Newtonian fluid and a linearized elastic solid. At least three different macroscopic models can be derived from this microscale formulation, depending on the assumptions on the fluid-structure interface. The first two are the well-known linear poroelasticity model [M. Biot, J. Appl. Phys., 12 (1941), pp. 155-164] and its nonlinear extension [C. K. Lee and C. C. Mei, Int. J. Eng. Sci., 35 (1997), pp. 329-352]. Both are derived under the assumption of small (at pore level) displacements of the interface with the difference that the first model excludes pore scale rigid body motions, while the second one accounts for them. A third macroscopic model is explained below. It concerns a particular case, namely, a porous medium formed by long parallel channels with thick elastic walls. An asymptotic solution to the FSI problem is derived for such a geometry, allowing finite (at pore scale) displacements for the interfaces. A nonlinear Darcy-type upscaled equation for the averaged pressure is obtained. The cell problems for each of the above cases, as well as a numerical algorithm for solving these cell problems, are discussed. The microscale cell FSI problems are treated numerically by an iterative procedure which solves sequentially fluid and solid subproblems and couples them via the interface conditions. Numerical and asymptotic solutions are found to converge to each other, thus validating both the numerical solver and the analytical derivation.
\end{abstract}

Key words. fluid-structure interaction, deformable porous media, upscaling, linear elasticity, stokes, finite elements

AMS subject classifications. AU MUST PROVIDE

DOI. $10.1137 / 06067732 \mathrm{X}$

1. Introduction. Flows in rigid and in deformable porous media are an essential part of many engineering and environmental processes, such as groundwater flow, reservoir engineering, various filtering devices, catalytic converters, microfluidic sensors, etc. Many multiscale problems for slow flow in saturated rigid porous media or media with a linear elastic skeleton are well understood (see, e.g., [7, 44]), and the accumulated knowledge is widely used in practical applications. Flow in porous media was first studied experimentally by [17] in the simplest possible setting of a rigid skeleton. Proper upscaled models for Stokes flow based on the homogenization

* Received by the editors December 10, 2006; accepted for publication (in revised form) July 24, 2007; published electronically DATE. This work was made possible with the financial support of the Kaiserslautern Excellence Cluster Dependable Adaptive Systems and Mathematical Modeling (DASMOD), Kaiserslautern, Germany.

http://www.siam.org/journals/mms/x-x/67732.html

${ }^{\dagger}$ Fraunhofer Institut für Techno- und Wirtschaftsmathematik, D-67657 Kaiserslautern, Germany (iliev@itwm.fhg.de). This author was partially supported by project INTAS-30-50-4395.

$\ddagger$ Université de Lyon, Lyon, F-69003, France, and Université Lyon 1, Institut Camille Jordan, UFR Mathématiques, Site de Gerland, Bât. A, 50, avenue Tony Garnier, 69367 Lyon Cedex 07, France (mikelic@univ-lyon1.fr). This author was partially supported by the GDR MOMAS (Modélisation Mathématique et Simulations numériques liées aux problèmes de gestion des déchets nucléaires: 2439 - ANDRA, BRGM, CEA, EDF, CNRS) as a part of the project "Changements d' échelle danséla modélisation du transport multiphasique et réactif en milieux poreux : application aux milieux fracturés et aux argiles."

$\S$ Institute for Scientific Computation, Texas A\&M University, College Station, TX 77843 (ppopov@tamu.edu). 
method appeared later $[51,8,50]$ and rely on so-called unit cell solutions in order to derive a diffusion equation at the macroscale. Darcy-type models in rigid porous media have been extended to more general microscale flows such as faster laminar flows (based on Navier-Stokes equations) [22, 7, 42, 21, 11], flows of viscoelastic fluids [52], flows of generalized Newtonian fluids [38, 12, 13, 2], or slow laminar flow of incompressible fluid over and within a porous bed [32, 34].

Considering flows in rigid porous media as multiscale problems allows us not only to get a better understanding of the underlying processes but also provides practical approaches for determining the coefficients of the macroscopic equations. Under certain restrictions (e.g., periodicity or statistic homogeneity), one can calculate coefficients in macroscopic equations by solving so-called cell problems [44, 30]. Fore example, solving Stokes problem at pore (micro-) level allows us to calculate permeability at meso- scale, while solving Darcy problem at mesoscale allows to calculate permeability tensor at macroscale. Discussions on numerical methods in Stokes to Darcy upscaling can be found, for example, in [31, 46]. Proper cell problems in the meso- to macroscale upscaling, as well as numerical methods for solving such problems, can be found, for example, in $[55,47,1]$.

The case of a deformable porous medium is more complicated, and much research is still needed to better understand respective multiscale problems. At the microscopic (pore) level one has a deformable skeleton surrounded by fluid flow. There exists a wide range of macroscale equations, which are used in the simulation of industrial and environmental problems involving flow in deformable porous media (cf., e.g., [36] and the references therein). Most of these models are derived at macroscale in a semiempirical way, and the coefficients are usually fitted on the basis of measurements, when possible. Opposite to the above approach, our aim is to consider and analyze the flow in deformable porous media as a multiscale problem. Depending on the assumptions about the interface, we will distinguish three cases. Case A: Linear poroelasticity. In this case the strains in the solid are small, allowing for the solid to be described by the Lame equations, and the considerations are restricted to small (even at pore level) displacements. Case B: Nonlinear poroelasticity. The displacements here can be decomposed into a rigid body motion at pore level and small extra displacements. Case C: Nonlinear Darcy. Here we consider finite displacement for the interface at pore level. Let us briefly discuss the three cases.

Case A: Linear poroelasticity. Similar to the development of models for Darcy flows, the problem was first studied experimentally by Biot [9] and Bear [7], who formulated the macroscopic equations for the effective medium. The application of the asymptotic homogenization method $[8,50,6,56]$ has led to theoretical justification of Biot's equation $[3,50,15,43]$ along with appropriate cell problems from which the macroscopic parameters can be computed numerically. These derivations rely on infinitely small fluid-solid interface displacements relative to the pore size. This allows the application of interface conditions at the initial position of the fluid-solid interface, and important properties such as periodicity of the unit cell are preserved. A wide range of applications, for example, in soil consolidation, acoustics, tissue modeling, etc., fall within these limits. However, many other important engineering problems cannot be considered under such restrictions.

Case B: Nonlinear poroelasticity. Under more relaxed assumptions, Lee and Mei [35] have derived a nonlinear macroscopic governing equation by assuming periodic media and allowing the interface displacements to be of the same order as the pore size. It is also assumed that the total deformation of a unit cell can be decomposed into 
a rigid body motion of each unit cell after which the interface displacement becomes infinitely small in the new reference frame. Under certain symmetry assumptions of the unit cell prior to the deformation it is shown that macroscopic equations reduce to Biot's law.

Case C: Nonlinear Darcy. The most general and least studied problem of flow in deformable porous media occurs when due to problem parameters such as microscopic pressure, the fluid-solid interface deforms considerably at the pore level. In such cases the interface cannot be approximated by a rigid body motion of its initial position, and it is necessary to consider the fluid-structure interaction (FSI) problem at pore level as a problem with an unknown interface. This microscale FSI problem is nonlinear despite the fact that the solid and fluid are described by linear equations. To our knowledge, rigorous upscaling for such a case is not known, even in very simple geometries.

The main targets of this paper are to contribute to a better understanding of the upscaling for porous media flow problems falling into Case $C$ and to present a proper numerical procedure for solving cell problems associated with Case A, Case B, and Case C.

For Case $C$, we present and discuss analytical and numerical approaches for simple geometries. We restrict ourselves to such two-/three-dimensional microscale geometries, which in turn lead to one-dimensional macroscopic equations. Such porous media can be formed from long straight channels with thick deformable walls (see Figure 7), from channels with partially rigid/partial elastic walls (Figure 9), from channels with rigid walls in the presence of deformable obstacles, etc.

For Case $A$, the rigorous derivation via homogenization theory goes along the same lines as in the nonstationary case (see $[23,16,20,45])$ and relies on the 2-scale convergence. For the formal derivation using 2-scale expansions we refer the reader to $[15,50,3]$. Formal derivation of the homogenized equations for Case $B$ is in the article [35]. Rigorous justification of the result is still an open challenging problem. In Case $C$ we use a combination of the 2-scale expansion and singular perturbation with respect to the ratio between the width and the length of the channel. In this way an effective nonlinear PDE for the pressure is derived. We note that the permeability in the corresponding Darcy law depends on the pressure in a nonlinear way. The derived equation was validated in comparison with the results from a direct numerical simulation, i.e., from the solution of the microscopic FSI problem.

An advantage of the numerical procedure presented here for the microscopic FSI is that it can also be applied when the scale separation is impossible. That is, for certain geometries, this procedure can become a basis of an efficient iterative scheme between different scales. Such a procedure was successfully used in [18] in connection with a nonlinear diffusion equation.

The reminder of the paper is organized as follows. The microscopic FSI problem is described in the next section. In the third section, macroscopic models and cell problems corresponding to Cases $A$ and $B$ are recalled. In the fourth section, an asymptotic solution for a Case $C$ cell problem is derived. The fifth section presents the discretization and the numerical algorithm used to solve the FSI problems. Numerical results are summarized in the sixth section. Finally, some conclusions are drawn.

2. The microscopic governing equations. Before we present the fluid-structure problem at the microscale we begin with a brief summary of the notation used, the formulation of the fluid, and solid problems alone. Consider a continuum body at the pore level. The material points in the body are associated with points $\mathbf{p}$ in $\mathbb{R}^{3}$. A 
body is defined as an open subset $\Omega \subset \mathbb{R}^{3}$. We denote the reference configuration by $\Omega_{0}$, which represents the body (solid or fluid) before the deformation has begun, and the deformed configuration by $\Omega$. We will use $\mathbf{p}$ to denote material coordinates and $\mathbf{x}$ to denote spatial ones.

In a FSI problem one has both a solid and a fluid which in this paper will be referred to as phases. We will need several basic concepts on the kinematics of continuum motion which are relevant to both the fluid and solid phase. The notation followed is the one from Gurtin [28], and the reader is referred to this classical text for more details. Consider a continuous, reversible deformation (cf., e.g., [28, 40])

$$
\mathbf{x}=x(\mathbf{p}),
$$

so that the reference and deformed configurations (of either the fluid or solid phase) are linked by $\Omega=\mathbf{x}\left(\Omega_{0}\right)$. The deformation gradient is denoted by $\mathbf{F}$ :

$$
\mathbf{F}(\mathbf{p})=\nabla \mathbf{x}(\mathbf{p}) \text {. }
$$

The deformation of solids is naturally described in the reference configuration, and the appropriate stress measure [28, Chapter V, pp. 97-114 ] in that case is the first Piola-Kirchhoff stress tensor $\mathbf{S}(\mathbf{p})$, a material field. ${ }^{1}$ The motion of fluids is naturally described by the Cauchy stress tensor $\mathbf{T}(\mathbf{x})$, a spatial field. The two are related by the identity $[28,40]$

$$
\mathbf{S}(\mathbf{p})=\operatorname{det}(\mathbf{F}(\mathbf{p})) \mathbf{T}(\mathbf{x}(\mathbf{p})) \mathbf{F}^{-T}(\mathbf{p}) .
$$

Finally, in order to simplify notation throughout the paper, it is convenient to introduce the symmetric part of the gradient operator:

$$
\mathbf{e}(\mathbf{w})=\frac{1}{2}\left(\nabla \mathbf{w}+(\nabla \mathbf{w})^{T}\right)
$$

for some field $\mathbf{w}$ (spatial or material). The solid and fluid problems, and the coupling interface conditions, are presented in the next three subsections.

2.1. Solid. Let $\mathbf{u}(\mathbf{p})$ be the displacements in the solid domain,

$$
\mathbf{u}(\mathbf{p})=\mathbf{x}(\mathbf{p})-\mathbf{p}
$$

and let $\mathbf{E}(\mathbf{p})$ be the usual infinite small strain [28]:

$$
\mathbf{E}(\mathbf{p})=\mathbf{e}(\mathbf{u})=\frac{1}{2}\left(\nabla \mathbf{u}(\mathbf{p})+\nabla \mathbf{u}(\mathbf{p})^{T}\right) .
$$

We restrict our attention to linearized elastic solids only, that is, solids for which

$$
\mathbf{S}=\mathrm{C}: \mathbf{E}
$$

where $\mathrm{C}$ is a fourth order elasticity tensor which defines the linear elastic solid, and $\mathrm{C}$ : $\mathbf{E}$ is well established in the mechanics literature notation for the double contraction,

\footnotetext{
${ }^{1} \mathrm{~A}$ material field is a scalar, vector, or tensor field which is specified on the reference configuration that is, it is a function of p. A spatial field is defined on the deformed configuration; that is, it is a function of $\mathbf{x}$. All derivatives are implicitly assumed over the appropriate variable. In that respect, a more detailed, but unnecessary, notation would be $\nabla_{\mathbf{x}} \mathbf{F}(\mathbf{x})$ instead of $\nabla \mathbf{F}(\mathbf{x})$ and $\nabla_{\mathbf{p}} \mathbf{T}(\mathbf{p})$ instead of $\nabla \mathbf{T}(\mathbf{p})$, etc.
} 
$\mathrm{C}: \mathbf{E}=\mathrm{C}^{i j m n} \mathbf{E}_{m n} \mathbf{g}_{\mathbf{i}} \oplus \mathbf{g}_{\mathbf{j}}$. The constitutive relation (2.6) is known as the generalized Hooke law. For a formal definition of the tensor C, as well as various other issues concerning this constitutive relation, the reader is referred to the classical work of Gurtin [28].

Then, given a body force $\mathbf{b}_{0}$ in the reference configuration, the boundary value problem for a linear elastic solid is stated (in the reference configuration) as follows: Find $\mathbf{u}(\mathbf{p})$ such that

$$
\nabla \cdot(\mathbf{C}: \mathbf{E})+\mathbf{b}_{0}=\mathbf{0} \quad \text { in } \Omega_{0},
$$

with Dirichlet

$$
\mathbf{u}=\hat{\mathbf{u}} \quad \text { on } \Gamma_{0}^{D},
$$

and/or Neumann

$$
\mathbf{S n}_{0}=\hat{\mathbf{s}} \quad \text { on } \Gamma_{0}^{N}
$$

boundary data, with the usual conditions $\Gamma_{0}^{D} \cap \Gamma_{0}^{N}=\varnothing$ and $\Gamma_{0}^{D} \cup \Gamma_{0}^{N}=\Gamma_{0}$.

As will be seen in section 2.3, a traction interface condition similar to (2.9) will be given on the part of the solid boundary which is an interface with the fluid.

2.2. Newtonian fluid at low Reynolds number. Newtonian fluids are best described using spatial fields. For stationary problems (the spatial description of all involved quantities is time independent), one has a velocity $\mathbf{v}(\mathbf{x})$ and, correspondingly, the symmetric part of the velocity gradient, namely, the stretching tensor $\mathbf{D}(\mathbf{x})$ given by

$$
\mathbf{D}(\mathbf{x})=\mathbf{e}(\mathbf{v})=\frac{1}{2}\left(\nabla \mathbf{v}(\mathbf{x})+\nabla \mathbf{v}(\mathbf{x})^{T}\right) .
$$

By definition, a Newtonian fluid is one for which

$$
\mathbf{T}=-p \mathbf{I}+2 \mu \mathbf{D},
$$

where $\mu$ is the absolute viscosity of the fluid. ${ }^{2}$ The fluid must satisfy conservation of mass,

$$
\nabla \cdot \mathbf{v}=\mathbf{0},
$$

and conservation of momentum. For a slow moving fluid, conservation of momentum reads

$$
-\mu \Delta \mathbf{v}+\nabla p=\mathbf{b},
$$

where $\mathbf{b}$ is a distributed body force (per unit volume) acting on the fluid.

2.3. The coupled FSI problem. Consider now the stationary fluid-structure problem (Figure 1) in the deformed configuration $\Omega=\Omega^{f} \cup \Omega^{s}$, where the fluid occupies $\Omega^{f}$, the solid occupies $\Omega^{s}$, and $\Omega^{f} \cap \Omega^{s}=\emptyset$. The part of the boundary shared between the fluid and the solid is denoted by $\Gamma^{I}=\partial \Omega^{f} \cap \partial \Omega^{s}$. Further, only open flow problems are considered, that is, ones for which there is a nonempty part of

\footnotetext{
${ }^{2}$ To be precise, this is a definition of a Newtonian fluid which is also independent under a change in observer. (Cf., e.g., [28, pp. 147-151].)
} 


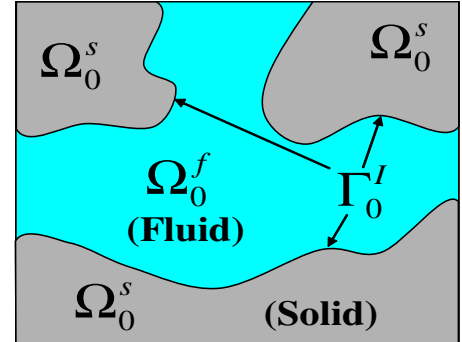

(a) Reference configuration

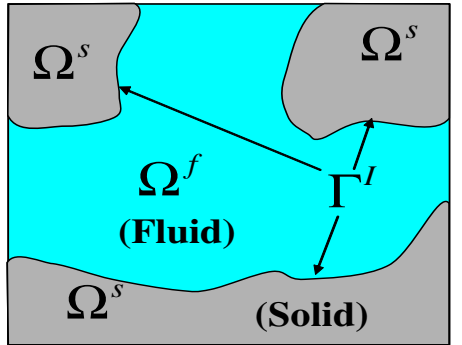

(b) Deformed configuration

FIG. 1. Schematic of the fluid and structure domains.

the boundary $\partial \Omega \backslash \Gamma^{I}$ through which fluid is allowed to pass. It is also assumed that the deformation $\mathbf{x}(\mathbf{p})$ is such that contact problems or breakup of the boundary do not occur. There are two conditions on the interface. The first is kinematic compatibility; that is, the velocity of the fluid on the interface should be equal to the velocity of the interface itself. This, for a stationary problem, implies that

$$
\mathbf{v}=\mathbf{0} \text { on } \Gamma^{I} .
$$

The second condition is continuity of tractions, namely (cf., e.g., [35]),

$$
\mathbf{T}^{f} \mathbf{n}=\mathbf{T}^{s} \mathbf{n} \quad \text { on } \Gamma^{I},
$$

where $\mathbf{n}=\mathbf{n}^{s}$ and $\mathbf{n}^{s}$ is the outward normal to the solid domain. The stress $\mathbf{T}^{f}$ in the fluid is given by (2.11). The Cauchy stress $\mathbf{T}^{s}$ can be expressed in terms of the Piola-Kirchhoff stress using (2.2), which together with Hooke's law (2.6) implies that

$$
-p \mathbf{n}+2 \mu \mathbf{D n}=\operatorname{det}(\mathbf{F})^{-1}(\mathbf{C}: \mathbf{E}) \mathbf{F}^{T} \mathbf{n} \quad \text { on } \Gamma^{I} .
$$

The stationary FSI problem therefore consists of finding the interface between the two domains, a velocity, pressure, and displacements which solve the Stokes equations (2.12), (2.13) and Lamé equation (2.7), respectively, and also satisfy the interface conditions (2.14) and (2.16). More formally, the FSI boundary value problem is summarized below in terms of the unknowns $\Gamma^{I}, \mathbf{v}, p$, and $\mathbf{u}$ : Find $\Gamma^{I}, \mathbf{v}, p$, and $\mathbf{u}$ such that

$$
\begin{aligned}
& \Gamma^{I}=\left\{\mathbf{p}+\mathbf{u}(\mathbf{p}) \mid \forall \mathbf{p} \in \Gamma_{0}^{I}\right\}, \\
& -\mu \Delta \mathbf{v}+\nabla p=\mathbf{b} \quad \text { in } \Omega^{f}, \\
& \nabla \cdot \mathbf{v}=\mathbf{0} \quad \text { in } \Omega^{f}, \\
& -\nabla \cdot(\mathbf{C}: \mathbf{E})=\mathbf{b}_{0} \quad \text { in } \Omega_{0}^{s} \text {, } \\
& \operatorname{det}(\nabla \mathbf{u}+\mathbf{I})(-p \mathbf{I}+2 \mu \mathbf{D}(\mathbf{x}(\mathbf{p})))(\nabla \mathbf{u}+\mathbf{I})^{-T} \mathbf{n}_{0}=(\mathbf{C}: \mathbf{E}) \mathbf{n}_{0} \text { on } \Gamma_{0}^{I},
\end{aligned}
$$

and $\mathbf{v}$ satisfies the kinematic interface condition (2.14); in addition $\mathbf{v}, p$, and $\mathbf{u}$ should also satisfy any boundary conditions that might be specified on $\partial \Omega \backslash \Gamma^{I}$. Equation (2.19) is the continuity of tractions (2.16) expressed on the reference position $\Gamma_{0}^{I}$ of 


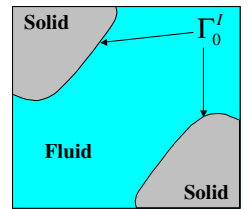

(a)

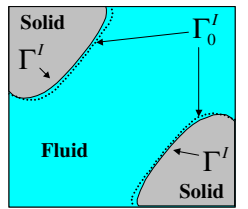

(b)

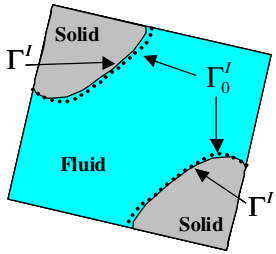

(c)

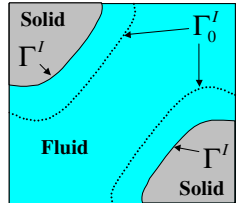

(d)

FIG. 2. Several possible types of FSI problems at the pore level. The reference configuration (a), infinitesimal displacements of the representative element of volume which corresponds to Biot's model (b), infinitesimal displacements superimposed on a rigid body motion (c), and a general case (d) of large pore-level interface displacements.

the interface. Observe that the position of the interface is part of the boundary value problem, and the solid-fluid coupling term (2.19) makes it a nonlinear one. Note that since only open flow problems are considered, there is no conservation of volume involved. $^{3}$

It should be noted that a linear constitutive relationship for the solid imposes a certain restriction on the deformation gradient $\mathbf{F}$ in the solid. The constitutive relation (2.6) provides an accurate description of a hyperelastic material [28] if there are no large rotations involved. However, this relation does not preclude the analysis of large pore-level deformation of the fluid domain and related consequences when upscaling the flow in such media - the deformation in the solid can easily be described by a linearized relationship, and yet the corresponding deformation of the fluid part of the body at the microscale can be significant. Examples of this type can be found in section 6 . On the other hand, the use of the linear relationship significantly simplifies the asymptotic analysis of section 4 .

3. Upscaling of deformable porous media. As was already mentioned in the introduction, at least three macroscopic models can be derived starting from the microscopic model formulated above. Let us briefly recall them. Depending on the assumptions about the interface, these are linear poroelasticity, nonlinear poroelasticity, and nonlinear Darcy models. In this section we will briefly discuss the first two cases; the third one will be considered in detail in the next section.

In the classical linear poroelasticity it is assumed that the solid is governed by the linearized constitutive relationship (2.6). The form of the homogenized equations depends on the magnitude of the displacements of the microscopic interface $\Gamma_{0}^{I}$. If one assumes infinitesimal displacements (Figure 2(b)), the media is described by the classical Biot law (cf., e.g., [9, 35]). Biot's law for the quasi-steady-state regime is

\footnotetext{
${ }^{3}$ If the fluid domain $\Omega^{f}$ is completely surrounded by solid, due to the incompressible nature of the Stokes flow, one will have to consider only fluid motions which are volume preserving. In such cases the additional constraint $\operatorname{det}(\mathbf{F})=1$ is required (cf., e.g., $[28,26]$ ), which is a necessary and sufficient condition for the fluid motion to be volume preserving.
} 


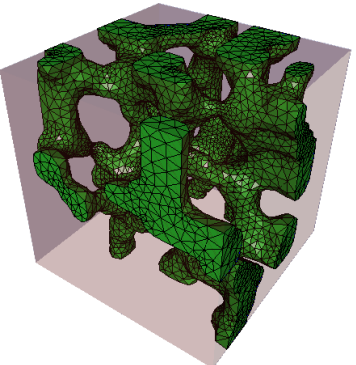

(a) REV geometry and solid domain mesh (interior)

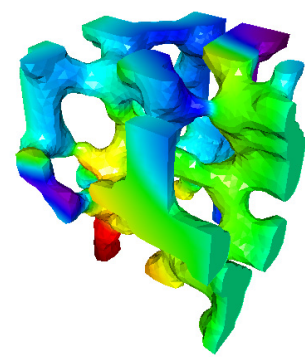

(b) Displacements, cell problem for the coupling term $\mathbf{A}^{*}$

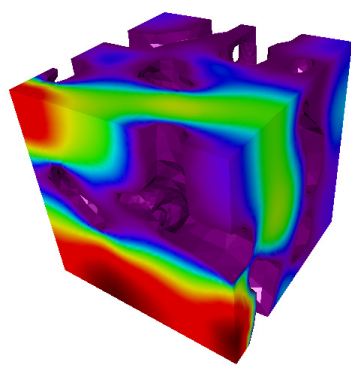

(c) Velocity component, one of the three Darcy cell problems for $\mathbf{K}^{*}$

FIG. 3. An example of typical Biot cell solutions for a nickel foam.

given by

$$
\begin{aligned}
\nabla \cdot\left(\mathbf{C}^{*}: \mathbf{e}(\langle\mathbf{u}\rangle)-\mathbf{A}^{*}\langle p\rangle\right) & =\mathbf{0}, \\
\nabla \cdot\left(\mathbf{K}^{*} \nabla\langle p\rangle-\rho \frac{\partial\langle\mathbf{u}\rangle}{\partial t}\right) & =\boldsymbol{\gamma}^{*}: \mathbf{e}\left(\frac{\partial\langle\mathbf{u}\rangle}{\partial t}\right)+\beta^{*} \frac{\partial\langle p\rangle}{\partial t} .
\end{aligned}
$$

Here $\langle p\rangle$ and $\langle\mathbf{u}\rangle$ are the macroscopic pressure and displacements. In the above equation the inertia effects in both the solid and fluid are disregarded. The interface, however, is assumed to change with time (at time scales much longer than what is required for transients to pass through either the solid and/or fluid), which leads to the time-dependent terms on the right-hand side of (3.2). These equations form the phenomenological theory of [9]. Later, they were rederived via asymptotic homogenization (cf., e.g., $[3,50])$. The reader is referred to [30] for a comprehensive review of several other forms of Biot's equations which incorporate various inertia and memory effects.

Equations (3.1) and (3.2) involve three different sets of cell problems defined on a representative element of volume (REV) and used to determine the effective properties $\mathbf{C}^{*}, \mathbf{A}^{*}, \mathbf{K}^{*}, \gamma^{*}$, and $\beta^{*}$. These cell problems are standard in the literature on linear poroelasticity, and their exact form can be found, for example, in [19, 35]. They are typically solved numerically, as in the example of a nickel foam, shown in Figure 3. The coefficient $C^{*}$ is simply the average macroscopic elastic properties of the porous skeleton, while $\mathbf{K}^{*}$ is the Darcy permeability for a rigid skeleton. The fluid-solid coupling coefficient $\mathbf{A}^{*}$ gives the solid stress due to a unit pressure on the interface. Observe that in the stationary case, the time derivatives of the displacement will disappear from (3.2), and it will decouple from (3.1). In that case one simply solves the linear Darcy flow (3.2) and substitutes the macroscopic pressure $\langle p\rangle$ into the elasticity equation (3.1), which can then be solved to obtain the average displacements $\langle\mathbf{u}\rangle$.

Various extensions of the linear poroelasticity model have been proposed, based on less restrictive assumptions about the displacements of the interface $\Gamma^{I}$. Lee and Mei [35] have recently obtained a generalization of Biot's equations by assuming that the cell displacement can be decomposed into a rigid body motion plus infinitely small deformation (Figure 2(c)) and that this rigid body motion is of the same order as the 
cell size. Using asymptotic expansions, the following nonlinear Biot-type equation on the macroscale was obtained:

$$
\nabla \cdot\left(\mathbf{C}^{*}: \mathbf{e}(\langle\mathbf{u}\rangle)-\langle\mathbf{A}\rangle\langle p\rangle\right)=C\left(\mathbf{G}^{*}: \mathbf{e}(\langle\mathbf{u}\rangle)+\boldsymbol{\alpha}^{*}\langle p\rangle\right): \nabla\langle\mathbf{u}\rangle,
$$

$\nabla \cdot\left(\mathbf{K}^{*} \nabla\langle p\rangle-\rho \frac{\partial\langle\mathbf{u}\rangle}{\partial t}\right)=\gamma^{*}: \mathbf{e}\left(\frac{\partial\langle\mathbf{u}\rangle}{\partial t}\right)+\beta^{*} \frac{\partial\langle p\rangle}{\partial t}+C(\langle\mathbf{J}\rangle: \mathbf{e}(\langle\mathbf{u}\rangle)+\langle\mathbf{M}\rangle\langle p\rangle) \nabla\langle p\rangle$.

Here $C, \mathbf{G}^{*}, \mathbf{J}^{*}$, and $\mathbf{M}^{*}$ are four additional macroscopic parameters. The reader is referred to [35] for details on the derivation of these macroscopic parameters and the means for their computation. Note that the left-hand side of (3.3) and (3.4) is the same as in Biot's equations and the rigid body motion of the REV introduces convective-like terms only at the right-hand side.

The combination of poroelasticity with large pore-level deformation (Figure 2(d)) is a complex problem. The first step in deriving upscaled models is to identify the relevant macroscopic upscaling parameters on a simple geometry. Traditionally, models such as Darcy and Biot have first been based on experimental observations; then solutions for the microscopic equations were obtained in channel-like geometries. Later the macroscopic equations were derived in general pore geometries using asymptotic expansions with well-defined cell problems. In the next section an asymptotic solution is derived for a fluid channel, sandwiched between two elastic slabs, undergoing large interface displacements. The resulting equation can be viewed as a one-dimensional, nonlinear Darcy flow.

4. An asymptotic solution for an elastic channel. In this section we consider the stationary laminar flow of incompressible Newtonian fluid through a twodimensional channel with linearly elastic walls. The reference geometry of the channel is shown in Figure 4 . Denote by $L$ the length of the channel, by $\ell$ the half of the channel width in the undeformed state, and by $\delta$ the thickness of the walls in the undeformed state. It is assumed that the channel is long, compared to its height; that is, the parameter $\varepsilon$

$$
\varepsilon=\frac{\ell}{L}
$$

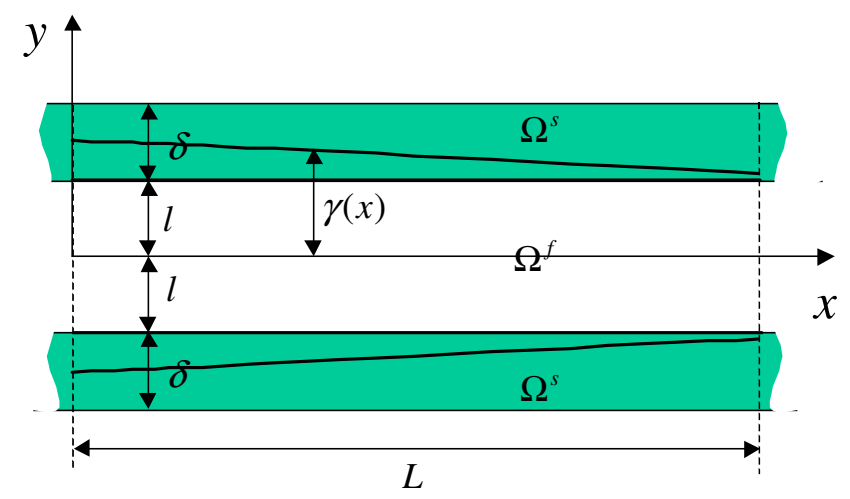

FIG. 4. Schematic of a section of length L, a long elastic channel. The fluid is driven by the pressure gradient. 
is small. Further, it is assumed that $\ell \sim \delta$. The fluid is driven by a pressure gradient, and the outer walls of the channel are fixed. Due to symmetry, only the upper half of the fluid geometry will be considered; that is, the fluid and solid occupy

$$
\begin{aligned}
& \Omega_{0}^{f}=\{(x, y), 0<x<L, 0<y<\ell\}, \\
& \Omega_{0}^{s}=\{(x, y), 0<x<L, l<y<\ell+\delta\},
\end{aligned}
$$

respectively. Further, the height of the unknown boundary of the fluid-solid interface is denoted by $\gamma(x)$. As a result, the fluid in the deformed configuration will occupy

$$
\Omega^{f}=\{(x, y), 0<x<L, 0<y<\gamma(x)\}
$$

To derive an asymptotic solution to the FSI problem first, the system of equations (2.17)-(2.19) will be normalized for this particular geometry (section 4.1). Then, in section 4.2, a formal expansion of the field variables (pressure, velocity, and displacements) with respect to the small parameter $\varepsilon$ will be used to obtain an asymptotic solution of (2.17)-(2.19). This asymptotic solution will further be interpreted as a one-dimensional Darcy flow with a nonlinear permeability.

4.1. Dimensionless form. Consider the dimensionless variables

$$
\begin{array}{ll}
x=L \tilde{x}, y=\ell \tilde{y}, & p(x, y)=\bar{P} \tilde{p}(\tilde{x}, \tilde{y}), \\
v_{1}(x, y)=\bar{V}_{1} \tilde{v}_{1}(\tilde{x}, \tilde{y}), & v_{2}(x, y)=\bar{V}_{2} \tilde{v}_{2}(\tilde{x}, \tilde{y}), \\
u_{1}(x, y)=\bar{U}_{1} \tilde{u}_{1}(\tilde{x}, \tilde{y}), & u_{2}(x, y)=\bar{U}_{2} \tilde{u}_{2}(\tilde{x}, \tilde{y}) .
\end{array}
$$

In these notations the fluid (4.4) and solid (4.3) domain are given by

$$
\begin{aligned}
& \Omega_{f}=\{(\tilde{x}, \tilde{y}): 0<\tilde{x}<1, \quad 0<\tilde{y}<\tilde{\gamma}(\tilde{x})\}, \\
& \Omega_{s}=\left\{(\tilde{x}, \tilde{y}): 0<\tilde{x}<1, \quad \tilde{\gamma}(\tilde{x})<\tilde{y}<1+\frac{\delta}{\ell}\right\},
\end{aligned}
$$

respectively, where

$$
\tilde{\gamma}(\tilde{x})=\frac{\gamma(x)}{\ell}
$$

Further, we also have $(i=1,2)$ :

$$
\begin{aligned}
\frac{\partial p}{\partial x} & =\frac{\bar{P}}{L} \frac{\partial \tilde{p}}{\partial \tilde{x}}, & \frac{\partial p}{\partial y} & =\frac{\bar{P}}{\ell} \frac{\partial \tilde{p}}{\partial \tilde{y}} \\
\frac{\partial v_{i}}{\partial x} & =\frac{\bar{V}_{i}}{L} \frac{\partial \tilde{v}_{i}}{\partial \tilde{x}}, & \frac{\partial v_{i}}{\partial y} & =\frac{\bar{V}_{i}}{\ell} \frac{\partial \tilde{v}_{i}}{\partial \tilde{y}} \\
\frac{\partial u_{i}}{\partial x} & =\frac{\bar{U}_{i}}{L} \frac{\partial \tilde{u}_{i}}{\partial \tilde{x}}, & \frac{\partial u_{i}}{\partial y} & =\frac{\bar{U}_{i}}{\ell} \frac{\partial \tilde{u}_{i}}{\partial \tilde{y}}
\end{aligned}
$$

It is clear that the scaling parameters cannot be chosen independently. Below we will discuss the relations between different scaling parameters.

4.1.1. Dimensionless Stokes equations. We first rewrite the Stokes system (2.12), (2.13) with respect to dimensionless variables. With the help of (4.9) and 
(4.10) we get

$$
\begin{aligned}
-\left(\varepsilon^{2} \frac{\partial^{2} \tilde{v}_{1}}{\partial \tilde{x}^{2}}+\frac{\partial^{2} \tilde{v}_{1}}{\partial \tilde{y}^{2}}\right)+\varepsilon^{2} \frac{\bar{P} L}{\mu \bar{V}_{1}} \frac{\partial \tilde{p}}{\partial \tilde{x}} & =0 \\
-\left(\varepsilon^{2} \frac{\partial^{2} \tilde{v}_{2}}{\partial \tilde{x}^{2}}+\frac{\partial^{2} \tilde{v}_{2}}{\partial \tilde{y}^{2}}\right)+\varepsilon \frac{\bar{P} L}{\mu \bar{V}_{2}} \frac{\partial \tilde{p}}{\partial \tilde{y}} & =0 \\
\frac{\partial \tilde{v}_{1}}{\partial \tilde{x}}+\frac{\bar{V}_{2}}{\varepsilon \bar{V}_{1}} \frac{\partial \tilde{v}_{2}}{\partial \tilde{y}} & =0 .
\end{aligned}
$$

In general, velocity components and pressure cannot be scaled independently. We choose $\bar{V}_{1}$ in accordance with maximal velocity of Poiseuille flow in a rigid channel:

$$
\bar{V}_{1}=4 V_{1, \max } \ell^{2}
$$

The other two scales, $\bar{V}_{2}$ and $\bar{P}$, are expressed by $\bar{V}_{1}$. In our case, the $x$-derivative of the pressure is the driving force for the flow; therefore, we want to keep it of unit size. Thus, without loss of generality,

$$
\frac{\bar{P} L}{\mu \bar{V}_{1}} \varepsilon^{2}=1
$$

that is,

$$
\bar{P}=\frac{\mu \bar{V}_{1}}{L \varepsilon^{2}}=\frac{\mu 4 V_{1, \max } \ell^{2} L}{\ell^{2}}=4 \mu V_{1, \max } L
$$

Further, we want to have strong conservation of mass. Therefore, we require

$$
\frac{\bar{V}_{2}}{\varepsilon \bar{V}_{1}}=1 \text {, }
$$

which gives us

$$
\bar{V}_{2}=\varepsilon \bar{V}_{1}
$$

The Stokes system (4.12)-(4.14) can now be rewritten as

$$
\begin{aligned}
-\varepsilon^{2} \frac{\partial^{2} \tilde{v}_{1}}{\partial \tilde{x}^{2}}-\frac{\partial^{2} \tilde{v}_{1}}{\partial \tilde{y}^{2}}+\frac{\partial \tilde{p}}{\partial \tilde{x}} & =0, \\
-\varepsilon^{2} \frac{\partial^{2} \tilde{v}_{2}}{\partial \tilde{x}^{2}}-\frac{\partial^{2} \tilde{v}_{2}}{\partial \tilde{y}^{2}}+\varepsilon^{-2} \frac{\partial \tilde{p}}{\partial \tilde{y}} & =0, \\
\frac{\partial \tilde{v}_{1}}{\partial \tilde{x}}+\frac{\partial \tilde{v}_{2}}{\partial \tilde{y}} & =0 .
\end{aligned}
$$

Here we have used the fact that under the assumptions (4.16) and (4.18) we have

$$
\frac{\bar{P} L}{\mu \bar{V}_{2}} \varepsilon \frac{\partial \tilde{p}}{\partial \tilde{y}}=\frac{\mu \bar{V}_{1}}{\varepsilon^{2} L} \frac{L}{\mu \bar{V}_{2}} \varepsilon \frac{\partial \tilde{p}}{\partial \tilde{y}}=\frac{\bar{V}_{1}}{\varepsilon^{2} \varepsilon \bar{V}_{1}} \varepsilon \frac{\partial \tilde{p}}{\partial \tilde{y}}=\varepsilon^{-2} \frac{\partial \tilde{p}}{\partial \tilde{y}}
$$


4.1.2. Dimensionless elasticity problem. Let us now consider the elastic domain. We restrict our attention only to isotropic solids. For an isotropic material, the elasticity tensor (2.6) is necessarily expressed in terms of the two Lamé constants, $\lambda_{s}$ and $\mu_{s}$ (cf., e.g., $[40,28]$ ), and so the stress tensor reduces to

$$
\mathbf{S}=\lambda_{s} \operatorname{tr}(\mathbf{E}) \mathbf{I}+2 \mu_{s} \mathbf{E} .
$$

With the help of (4.11), $\mathbf{S}$ of (4.21) can be expressed in terms of the nondimensional variables $(4.7)$ as

$$
\mathbf{S}^{s}=\left[\begin{array}{rr}
\left(\lambda_{s}+2 \mu_{s}\right) \frac{\bar{U}_{1}}{L} \frac{\partial \tilde{u}_{1}}{\partial \tilde{x}}+\lambda_{s} \frac{\bar{U}_{2}}{l} \frac{\partial \tilde{u}_{2}}{\partial \tilde{y}} & \mu_{s}\left(\frac{\bar{U}_{1}}{l} \frac{\partial \tilde{u}_{1}}{\partial \tilde{y}}+\frac{\bar{U}_{2}}{L} \frac{\partial \tilde{u}_{2}}{\partial \tilde{x}}\right) \\
\mu_{s}\left(\frac{\bar{U}_{1}}{l} \frac{\partial \tilde{u}_{1}}{\partial \tilde{y}}+\frac{\bar{U}_{2}}{L} \frac{\partial \tilde{u}_{2}}{\partial \tilde{x}}\right) & \left(\lambda_{s}+2 \mu_{s}\right) \frac{\bar{U}_{2}}{l} \frac{\partial \tilde{u}_{2}}{\partial \tilde{y}}+\lambda_{s} \frac{\bar{U}_{1}}{L} \frac{\partial \tilde{u}_{1}}{\partial \tilde{x}}
\end{array}\right] .
$$

Further, assume that

$$
\bar{U}_{2}=\delta, \bar{U}_{1}=\varepsilon^{0} \bar{U}_{2}=\delta .
$$

Using this scaling for the displacements, the stress in the solid becomes

$$
\mathbf{S}^{s}=\frac{\delta}{\ell}\left[\begin{array}{rr}
\left(\lambda_{s}+2 \mu_{s}\right) \varepsilon \frac{\partial \tilde{u}_{1}}{\partial \tilde{x}}+\lambda_{s} \frac{\partial \tilde{u}_{2}}{\partial \tilde{y}} & \mu_{s} \frac{\partial \tilde{u}_{1}}{\partial \tilde{y}}+\mu_{s} \varepsilon \frac{\partial \tilde{u}_{2}}{\partial \tilde{x}} \\
\mu_{s} \frac{\partial \tilde{u}_{1}}{\partial \tilde{y}}+\mu_{s} \varepsilon \frac{\partial \tilde{u}_{2}}{\partial \tilde{x}} & \left(\lambda_{s}+2 \mu_{s}\right) \frac{\partial \tilde{u}_{2}}{\partial \tilde{y}}+\lambda_{s} \varepsilon \frac{\partial \tilde{u}_{1}}{\partial \tilde{x}}
\end{array}\right]
$$

Further, it is also necessary to write the system of elasticity equations (2.7) in nondimensional form. For an isotropic solid, and in the absence of a body force, it is easy to see that $(2.7)$ reduces to

$$
\begin{aligned}
& \varepsilon^{2} \delta\left(\lambda_{s}+2 \mu_{s}\right) \frac{\partial^{2} \tilde{u}_{1}}{\partial \tilde{x}^{2}}+\varepsilon \delta\left(\lambda_{s}+\mu_{s}\right) \frac{\partial^{2} \tilde{u}_{2}}{\partial \tilde{x} \partial \tilde{y}}+\delta \mu_{s} \frac{\partial^{2} \tilde{u}_{1}}{\partial \tilde{y}^{2}}=0, \\
& \varepsilon^{2} \delta \mu_{s} \frac{\partial^{2} \tilde{u}_{2}}{\partial \tilde{x}^{2}}+\varepsilon \delta\left(\mu_{s}+\lambda_{s}\right) \frac{\partial^{2} \tilde{u}_{1}}{\partial \tilde{x} \partial \tilde{y}}+\delta\left(\lambda_{s}+2 \mu_{s}\right) \frac{\partial^{2} \tilde{u}_{2}}{\partial \tilde{y}^{2}}=0 .
\end{aligned}
$$

4.2. Asymptotic expansion. Consider now an asymptotic expansion of the field variables with respect to the small parameter $\varepsilon$ :

$$
\begin{aligned}
\tilde{v}_{i} & =\tilde{v}_{i}^{0}+\varepsilon \tilde{v}_{i}^{1}+\varepsilon^{2} \tilde{v}_{i}^{2}+\cdots, \\
\tilde{p} & =\tilde{p}^{0}+\varepsilon \tilde{p}^{1}+\varepsilon^{2} \tilde{p}^{2}+\cdots, \\
\tilde{u}_{i} & =\tilde{u}_{i}^{0}+\varepsilon \tilde{u}_{i}^{1}+\varepsilon^{2} \tilde{u}_{i}^{2}+\cdots .
\end{aligned}
$$

4.2.1. Asymptotic expansion for Stokes system. Substituting these expansions into Stokes system (4.20) and collecting terms corresponding to different powers of $\varepsilon$, we get

$$
\begin{array}{ll}
\text { at order } \varepsilon^{-2}: & \frac{\partial \tilde{p}^{0}}{\partial \tilde{y}}=0, \\
\text { at order } \varepsilon^{-1}: & \frac{\partial \tilde{p}^{1}}{\partial \tilde{y}}=0 .
\end{array}
$$


Next, at order $\varepsilon^{0}$ we obtain

$$
\begin{aligned}
-\frac{\partial^{2} \tilde{v}_{1}^{0}}{\partial \tilde{y}^{2}}+\frac{\partial \tilde{p}^{0}}{\partial \tilde{x}} & =0, \\
-\frac{\partial^{2} \tilde{v}_{2}^{0}}{\partial \tilde{y}^{2}}+\frac{\partial \tilde{p}^{2}}{\partial \tilde{y}} & =0, \\
\frac{\partial \tilde{v}_{1}^{0}}{\partial \tilde{x}}+\frac{\partial \tilde{v}_{2}^{0}}{\partial \tilde{y}} & =0 .
\end{aligned}
$$

Hence, from (4.27) and (4.28) the first two terms in the pressure expansion are found to depend only on the $x$-coordinate:

$$
\tilde{p}^{0}=\tilde{p}^{0}(x), \quad \tilde{p}^{1}=\tilde{p}^{1}(x) .
$$

Next, (4.29a) is integrated with respect to $\tilde{y}$. The integration limits are from 0 (symmetry line) to $\tilde{y}$, and using the symmetry condition this yields

$$
\frac{\partial \tilde{v}_{1}^{0}}{\partial \tilde{y}}=\tilde{y} \frac{\partial \tilde{p}^{0}}{\partial \tilde{x}} .
$$

This equation, after another integration with respect to $\tilde{y}$, from $\tilde{y}$ to $\tilde{\gamma}(\tilde{x})$, becomes

$$
\tilde{v}_{1}^{0}(\tilde{x}, \tilde{\gamma}(\tilde{x}))-v_{1}^{0}(\tilde{x}, \tilde{y})=\frac{1}{2}\left(\tilde{\gamma}^{2}(\tilde{x})-\tilde{y}^{2}\right) \frac{\partial \tilde{p}^{0}}{\partial \tilde{x}} .
$$

In light of the no-slip boundary condition $\tilde{v}_{1}^{0}(\tilde{x}, \tilde{\gamma}(\tilde{x}))=0$, it reduces to

$$
\tilde{v}_{1}^{0}(\tilde{x}, \tilde{y})=-\frac{\tilde{\gamma}^{2}(\tilde{x})-\tilde{y}^{2}}{2} \frac{\partial \tilde{p}^{0}}{\partial \tilde{x}} .
$$

Equation (4.32) leads to the following expression for the $\tilde{x}$-derivative of $\tilde{v}_{1}^{0}$ :

$$
\frac{\partial \tilde{v}_{1}^{0}}{\partial \tilde{x}}=-\frac{\tilde{\gamma}^{2}(\tilde{x})-\tilde{y}^{2}}{2} \frac{\partial^{2} \tilde{p}^{0}}{\partial \tilde{x}^{2}}-\tilde{\gamma}(\tilde{x}) \frac{\partial \tilde{\gamma}(\tilde{x})}{\partial \tilde{x}} \frac{\partial \tilde{p}^{0}}{\partial \tilde{x}} .
$$

Integrating the continuity equation $(4.29 \mathrm{c})$ from 0 to $\tilde{\gamma}$, using the symmetry condition $v_{2}^{0}(\tilde{x}, 0)=0$, the interface condition $v_{2}^{0}(\tilde{x}, \tilde{\gamma})=0$, and (4.33), one obtains

$$
\begin{aligned}
0=\int_{0}^{\tilde{\gamma}} \frac{\partial \tilde{v}_{1}^{0}(\tilde{x}, s)}{\partial \tilde{x}} d s & =-\frac{1}{2} \frac{\partial^{2} \tilde{p}^{0}}{\partial \tilde{x}^{2}}\left(\tilde{\gamma}^{3}(\tilde{x})-\frac{\tilde{\gamma}^{3}(\tilde{x})}{3}\right)-\tilde{\gamma}^{2}(\tilde{x}) \frac{\partial \tilde{\gamma}(\tilde{x})}{\partial \tilde{x}} \frac{\partial \tilde{p}^{0}}{\partial \tilde{x}} \\
& =-\frac{\partial^{2} \tilde{p}^{0}}{\partial \tilde{x}^{2}} \frac{\tilde{\gamma}^{3}(\tilde{x})}{3}-\frac{1}{3} \frac{\partial \tilde{\gamma}^{3}(\tilde{x})}{\partial \tilde{x}} \frac{\partial \tilde{p}^{0}}{\partial \tilde{x}}=-\frac{1}{3} \frac{\partial}{\partial \tilde{x}}\left(\tilde{\gamma}^{3}(\tilde{x}) \frac{\partial \tilde{p}^{0}}{\partial \tilde{x}}\right) .
\end{aligned}
$$

Therefore, the following equation is obtained with respect to $p^{0}(x)$ :

$$
-\frac{\partial}{\partial \tilde{x}}\left(\frac{1}{3} \tilde{\gamma}^{3}(\tilde{x}) \frac{\partial \tilde{p}^{0}}{\partial \tilde{x}}\right)=0 .
$$

Observe that (4.34) can be interpreted as a Darcy flow in a one-dimensional porous medium. To do this, fix $\tilde{x}$ and define the $\tilde{y}$-average operator $\langle\cdot\rangle_{\tilde{y}}$ :

$$
\left.\langle\tilde{\phi}(\tilde{x}, \tilde{y})\rangle_{\tilde{y}}:=\frac{1}{2} \int_{-\tilde{\gamma}(\tilde{x})}^{\tilde{\gamma}(\tilde{x})} \tilde{\phi}(\tilde{x}, \tilde{y})\right) d y,
$$


where $\tilde{\phi}(\tilde{x}, \tilde{y})$ is a generic function. The average is with respect to the reference width of the fluid channel. Then, after taking the average of (4.32), one obtains

$$
\left\langle\tilde{v}_{1}(\tilde{x})\right\rangle=-\frac{1}{3} \tilde{\gamma}^{3}(\tilde{x}) \frac{\partial \tilde{p}^{0}}{\partial \tilde{x}} .
$$

One would now recognize that (4.34) is the conservation of mass for a flow with flux $\left\langle\tilde{v}_{1}(\tilde{x})\right\rangle$, driven by a pressure gradient $\frac{\partial \tilde{\tilde{p}}^{0}}{\partial \tilde{x}}$. Furthermore, the quantity $\tilde{K}$,

$$
\tilde{K}:=\tilde{K}(\tilde{\gamma}(\tilde{x}))=-\mu \frac{\left\langle\tilde{v}_{1}(\tilde{x})\right\rangle}{\partial_{\tilde{x}} \tilde{p}^{0}(\tilde{x})}=\frac{\mu}{3} \tilde{\gamma}^{3}(\tilde{x}),
$$

defined as the ratio of the mass flux and the pressure gradient, can be interpreted as permeability of the channel. The permeability is scaled, as usual, by the fluid viscosity, and so it does not depend on the fluid properties. In the rigid case $(\tilde{\gamma}(\tilde{x})=1)$ it will coincide with the standard Darcy permeability. When the channel is deformable, however, it is not constant but will depend on the third power of the channel opening. With the definition (4.37) in mind, (4.34) can be rewritten as

$$
-\frac{\partial}{\partial \tilde{x}}\left(\tilde{K}(\tilde{\gamma}(\tilde{x})) \frac{\partial \tilde{p}^{0}}{\partial \tilde{x}}\right)=0 .
$$

The remainder of this section is devoted to obtaining an expression for $\tilde{\gamma}$ in terms of $\tilde{p}^{0}$ which will close $(4.38)$, so that it can be solved for $\tilde{p}^{0}$ with some appropriate boundary conditions. To do this the asymptotic expansion for elasticity system is considered next.

4.2.2. Asymptotic expansion for elasticity system. Now the asymptotic expansion for $u_{1}, u_{2}$ is substituted into the elasticity system (4.23), (4.24). From the first elasticity equation $(4.23)$, at order $\varepsilon^{0}$, one obtains

$$
\mu_{s} \frac{\partial^{2} \tilde{u}_{1}^{0}}{\partial \tilde{y}^{2}}=0
$$

After integration with respect to $\tilde{y}$, this equation becomes

$$
\mu_{s} \frac{\partial \tilde{u}_{1}^{0}}{\partial \tilde{y}}=c_{1}(\tilde{x})
$$

Integrating again from $\tilde{y}$ to $\left(1+\frac{\delta}{\ell}\right)$ and using the fact that $\tilde{u}_{1}^{0}=0$ at the upper boundary, one gets

$$
\tilde{u}_{1}^{0}(\tilde{x}, \tilde{y})=-\frac{\left(1+\frac{\delta}{\ell}-\tilde{y}\right)}{\mu_{s}} c_{1}(\tilde{x}) .
$$

Similarly, by considering the second elasticity equation (4.24) from the elasticity system at order $\varepsilon^{0}$, integrating twice with respect to $\tilde{y}$, and using the boundary condition $\tilde{u}_{2}^{0}=0$, one gets

$$
\tilde{u}_{2}^{0}(\tilde{x}, \tilde{y})=-\frac{\left(1+\frac{\delta}{\ell}-\tilde{y}\right)}{\lambda_{s}+2 \mu_{s}} c_{2}(\tilde{x}) .
$$


4.2.3. Expansion of solid-fluid interface condition. The last step in obtaining an expression for the boundary $\tilde{\gamma}(\tilde{x})$, and thus a closed-form expression for the upscaled equation (4.38), is to consider the interface condition (2.19) for the continuity of the normal component of the stress tensor. Consider first the fluid stress $\mathbf{T}^{f}$. Using (4.9) and (4.10) as well as (2.10) and (2.11), $\mathbf{T}^{f}$ becomes

$$
\mathbf{T}^{f}=\bar{P}\left[\begin{array}{cc}
2 \varepsilon^{2} \frac{\partial \tilde{v}_{1}}{\partial \tilde{x}}-\tilde{p} & \varepsilon \frac{\partial \tilde{v}_{1}}{\partial \tilde{y}}+\varepsilon^{3} \frac{\partial \tilde{v}_{2}}{\partial \tilde{x}} \\
\varepsilon \frac{\partial \tilde{v}_{1}}{\partial \tilde{y}}+\varepsilon^{3} \frac{\partial \tilde{v}_{2}}{\partial \tilde{x}} & 2 \varepsilon^{2} \frac{\partial \tilde{v}_{2}}{\partial \tilde{y}}-\tilde{p}
\end{array}\right] .
$$

Next, the expansions for velocity and pressure from (4.25) are substituted into (4.42) to obtain

$$
\mathbf{T}^{f}=\frac{\mu \bar{V}_{1} L}{\ell^{2}}\left[\begin{array}{lr}
-\tilde{p}^{0}(\tilde{x}) & 0 \\
0 & -\tilde{p}^{0}(\tilde{x})
\end{array}\right]+\mathcal{O}(\varepsilon) .
$$

Second, consider the solid stresses $\mathbf{S}^{s}$ and substitute the asymptotic expansion (4.26) for $\tilde{u}_{1}, \tilde{u}_{2}$ into (4.22) to obtain, at order $\varepsilon^{0}$,

$$
\mathbf{S}^{s, 0}=\frac{\delta}{\ell}\left[\begin{array}{cr}
\lambda_{s} \frac{\partial \tilde{u}_{2}^{0}}{\partial \tilde{y}} & \mu_{s} \frac{\partial \tilde{u}_{1}^{0}}{\partial \tilde{y}} \\
\mu_{s} \frac{\partial \tilde{u}_{1}^{0}}{\partial \tilde{y}} & \left(\lambda_{s}+2 \mu_{s}\right) \frac{\partial \tilde{u}_{2}^{0}}{\partial \tilde{y}}
\end{array}\right] .
$$

Then $\tilde{u}_{1}^{0}, \tilde{u}_{2}^{0}$ as given by (4.40) and (4.41), respectively, are substituted into the last equation to obtain

$$
\mathbf{S}^{s, 0}=\frac{\delta}{\ell}\left[\begin{array}{ll}
\frac{\lambda_{s}}{\lambda_{s}+2 \mu_{s}} c_{2}(\tilde{x}) & c_{1}(\tilde{x}) \\
c_{1}(\tilde{x}) & c_{2}(\tilde{x})
\end{array}\right] .
$$

Using this last result, the normal component of the zeroth order term for the stress tensor can be calculated at the interface. This is done at the reference configuration, for which the normal is simply $\mathbf{n}_{0}=\mathbf{e}_{2}$ :

$$
\mathbf{S}^{s, 0} \mathbf{n}_{0}=\frac{\delta}{\ell}\left(c_{1}(\tilde{x}) \mathbf{e}_{1}+c_{2}(\tilde{x}) \mathbf{e}_{2}\right) .
$$

Note that the last equation cannot be compared directly with (4.43), as the first is in Lagrangian coordinates, while the former is in Eulerian coordinates. However, the point $(\tilde{x}, 1)$, at the interface in the reference configuration, corresponds to the interface point $\left(\tilde{x}_{E}, \tilde{\gamma}\left(\tilde{x}_{E}\right)\right)$ in the Eulerian formulation, where

$$
\tilde{x}_{E}=\tilde{x}+\tilde{u}_{1}^{0}(\tilde{x}) \quad \text { and } \quad \tilde{\gamma}\left(\tilde{x}_{E}\right)=1+\tilde{u}_{2}^{0}(\tilde{x}) .
$$

The fluid stress force at the interface in the reference configuration is given by the expression at the left-hand side in the formula (2.19). To use (2.19) one needs the leading order of the deformation gradient:

$$
\mathbf{F}=\nabla \mathbf{u}+\mathbf{I}=\left(\begin{array}{cc}
1+\frac{\partial u_{1}}{\partial x} & \frac{\partial u_{1}}{\partial y} \\
\frac{\partial u_{2}}{\partial x} & 1+\frac{\partial u_{2}}{\partial y}
\end{array}\right)=\left(\begin{array}{cc}
1+\varepsilon \frac{\partial \tilde{u}_{1}}{\partial \tilde{x}} & \frac{\delta}{l} \frac{\partial \tilde{u}_{1}}{\partial \tilde{y}} \\
\varepsilon \frac{\partial \tilde{u}_{2}}{\partial \tilde{x}} & 1+\frac{\delta}{l} \frac{\partial \tilde{u}_{2}}{\partial \tilde{y}}
\end{array}\right) ;
$$


therefore, $\mathbf{F}=\mathbf{F}^{0}+\mathcal{O}(\varepsilon)$, where

$$
\mathbf{F}^{0}=\left(\begin{array}{cc}
1 & \frac{\delta}{l} \frac{\partial \tilde{u}_{1}^{0}}{\partial \tilde{y}} \\
0 & 1+\frac{\delta}{l} \frac{\partial \tilde{u}_{2}^{0}}{\partial \tilde{y}}
\end{array}\right) \quad \text { and } \quad\left(\mathbf{F}^{0}\right)^{-T}=\left(1+\frac{\delta}{l} \frac{\partial \tilde{u}_{2}^{0}}{\partial \tilde{y}}\right)^{-1}\left(\begin{array}{cc}
1+\frac{\delta}{l} \frac{\partial \tilde{u}_{2}^{0}}{\partial \tilde{y}} & 0 \\
-\frac{\delta}{l} \frac{\partial \tilde{u}_{1}^{0}}{\partial \tilde{y}} & 1
\end{array}\right) .
$$

The insertion of (4.43), (4.44), and (4.47) into (2.19) now results in

$$
-\bar{P} \tilde{p}^{0}(\tilde{x}) \mathbf{e}_{2}=\frac{\delta}{\ell}\left(c_{1}(\tilde{x}) \mathbf{e}_{1}+c_{2}(\tilde{x}) \mathbf{e}_{2}\right)+\mathcal{O}(\varepsilon)
$$

A direct consequence of (4.48) is that we do not have a longitudinal displacement at zero order, i.e., $\tilde{u}_{1}^{0}=c_{1}(\tilde{x})=0$. This result greatly simplifies the link (4.45) between the Lagrangian and the Eulerian formulations. Now $(\tilde{x}, 1)$ transforms into $(\tilde{x}, \tilde{\gamma}(\tilde{x}))$, and hence

$$
c_{2}(\tilde{x})=-\bar{P} \frac{l}{\delta} \tilde{p}^{0}(\tilde{x}) .
$$

Next, with the help of (4.41), the vertical displacement is found to be

$$
\tilde{u}_{2}^{0}(\tilde{x}, \tilde{y})=\frac{1+\frac{\ell}{\delta}(1-\tilde{y})}{\lambda_{s}+2 \mu_{s}} \bar{P} \tilde{p}^{0}(\tilde{x}) .
$$

Equation (4.49) leads to the following expression for the interface position:

$$
\tilde{\gamma}(\tilde{x})=1+\tilde{u}_{2}^{0}(\tilde{x}, 1)=1+\frac{\bar{P}}{\lambda_{s}+2 \mu_{s}} \tilde{p}^{0}(\tilde{x}) .
$$

Equivalently, in dimensional variables,

$$
\gamma(x)=\ell+\frac{\ell}{\lambda_{s}+2 \mu_{s}} p^{0}(x) .
$$

4.2.4. Permeability of a long elastic channel. When the formula for $\gamma(x)$ given in (4.50) is substituted into (4.37), the permeability $\tilde{K}$ is found to be

$$
\tilde{K}=\tilde{K}\left(\tilde{p}^{0}(\tilde{x}), \tilde{x}\right)=\frac{\mu}{3} \tilde{\gamma}^{3}(\tilde{x})=\frac{\mu}{3}\left(1+\frac{\bar{P}}{\lambda_{s}+2 \mu_{s}} \tilde{p}^{0}(\tilde{x})\right)^{3} .
$$

The last expression is a function only of the pressure $\tilde{p}^{0}$, which implies that upscaled Darcy equation (4.38) can now be closed, and so it is fully defined in terms of $\tilde{p}^{0}$ :

$$
-\frac{\partial}{\partial \tilde{x}}\left(\frac{1}{3}\left(1+\frac{\bar{P}}{\lambda_{s}+2 \mu_{s}} \tilde{p}^{0}(\mathbf{x})\right)^{3} \frac{\partial \tilde{p}^{0}(\tilde{x})}{\partial \tilde{x}}\right)=0 .
$$

This section is concluded by giving the dimensional form of the permeability $\tilde{K}$ and the upscaled equation (4.38). Let the generic function $\tilde{\phi}$ (cf. (4.35)) be given in dimensional form $\phi(x, y)=\bar{\Phi} \tilde{\phi}(\tilde{x}, \tilde{y})$ with some scaling factor $\bar{\Phi}$. It is straightforward to check that the $y$-average operator $\langle\cdot\rangle_{y}$ satisfies

$$
\langle\phi(x, y)\rangle_{y}:=\frac{1}{2 l} \int_{-\gamma(x)}^{\gamma(x)} \phi(x, y) d y=\frac{1}{2} \int_{-\tilde{\gamma}(\tilde{x})}^{\tilde{\gamma}(\tilde{x})} \phi(\tilde{x}, \tilde{y}) d \tilde{y}=\bar{\Phi}\langle\tilde{\phi}(\tilde{x}, \tilde{y})\rangle_{\tilde{y}} .
$$


Therefore, the permeability $K$, in dimensional form, is obtained, with the help of (4.6), (4.9), (4.54), (4.37), (4.8), and (4.16), as

$$
K:=-\mu \frac{\left\langle v_{1}(x)\right\rangle}{\partial_{x} p^{0}(x)}=-\frac{\mu \bar{V}_{1} L}{\bar{P}} \frac{\left\langle\tilde{v}_{1}(\tilde{x})\right\rangle}{\partial_{\tilde{x}} \tilde{p}^{0}(\tilde{x})}=\frac{\mu \bar{V}_{1} L}{\bar{P}} \frac{1}{3} \tilde{\gamma}^{3}(\tilde{x})=\frac{\mu \bar{V}_{1} L}{\bar{P} l^{2}} \frac{1}{3 l} \gamma^{3}(x)=\frac{1}{3 l} \gamma^{3}(x) .
$$

Now, using (4.51), $K$ is expressed as a function of pressure:

$$
K=K\left(p^{0}(x), x\right)=\frac{1}{3 \ell}\left(l+\frac{l}{\lambda_{s}+2 \mu_{s}} p^{0}(\mathbf{x})\right)^{3}=\frac{\ell^{2}}{3}\left(1+\frac{p^{0}(\mathbf{x})}{\lambda_{s}+2 \mu_{s}}\right)^{3},
$$

and the upscaled equation (4.53) (or, equivalently, (4.38)) in dimensional variables becomes

$$
-\frac{\partial}{\partial x}\left(K\left(p^{0}(x), x\right) \frac{\partial p^{0}(x)}{\partial x}\right)=0 \quad \text { in }(0, L) .
$$

Equations (4.57) and (4.56) form a closed, nonlinear system, which, along with some appropriate boundary conditions, for example, Dirichlet data,

$$
p^{0}(0)=P_{\text {left }}, \quad p^{0}(L)=P_{\text {right }},
$$

becomes a well-posed boundary value problem for the upscaled pressure $p^{0}$. It is important to emphasize that in deriving this PDE equation we do not pass to the limit when $\varepsilon=\ell / L \rightarrow 0$. Rather, we calculate the leading-order terms for the longitudinal velocity and for the pressure field.

It is also useful to give some physical interpretation to the system (4.56), (4.57). Observe that using the expression (4.56) along with the first equality in (4.55), we see that the effective velocity (in dimensional form) is

$$
\left\langle v_{1}(x)\right\rangle=-\frac{\ell^{2}}{3 \mu}\left(1+\frac{p^{0}(\mathbf{x})}{\lambda_{s}+2 \mu_{s}}\right)^{3} \frac{\partial p^{0}(x)}{\partial x} .
$$

This means that for a fixed pressure drop $\partial_{x} p^{0}(x)$, the ability of fluid to flow through a channel is proportional to a cubic function of the pressure $p^{0}(x)$ and to the reference channel half-width $\ell^{2}$ divided by the fluid viscosity $\mu$. In the situation of flow through a slit with rigid walls one has $\lambda_{s}, \mu_{s} \rightarrow \infty$; that is, the dependence on the pressure $p^{0}(x)$ disappears, and reduces to the classical case:

$$
\left\langle v_{1}(x)\right\rangle=-\frac{\ell^{2}}{3 \mu} \frac{\partial p^{0}(x)}{\partial x} .
$$

The difference comes from the proportionality factor $\left(1+\frac{p^{0}(\mathbf{x})}{\lambda_{s}+2 \mu_{s}}\right)^{3}$, which, in the case of elastic walls, is different from identity and could be important. Thus, the nonlinear filtration law (4.59) distinguishes the flow through an elastic channel and includes the rigid walls channel as a special limiting case.

As a final remark, if we model our porous medium as a network of parallel capillary tubes, then their number will be of order $\ell^{-2}$ and the mass flow will remain constant when the tube diameter tends to zero.

5. Discretization of the coupled FSI system. In this section we present a numerical method for the solution of the FSI problem. First, the continuum problems are recast in weak form in section 5.1 and then, in section 5.2, are discretized, giving rise to a nonlinear system of algebraic equations. Further, in section 5.3 an iterative procedure is presented, which solves the coupled FSI problem. It relies on the consecutive solution of solid and fluid subproblems. 
5.1. Weak form of the elasticity, Stokes, and FSI problems. Consider a generic (either fluid or solid) bounded Lipschitz domain $\Omega$, and let $(\cdot, \cdot)_{\Omega}$ be the usual inner product on $L^{2}(\Omega)$ and, as there is no chance of confusion, also the inner product on $\left[L^{2}(\Omega)\right]^{d}$, where $d=2,3$ is the size of the spatial dimension. Let $H^{m}(\Omega)$, $m= \pm 1$, be the standard Sobolev spaces and $L_{0}^{2}(\Omega)$ be the Hilbert space of functions in $L^{2}$ having zero mean. Also, denote by $H_{D}^{1}(\Omega) \subset H^{1}(\Omega)$ the subspace of functions in $H^{1}(\Omega)$ which vanish on part of the boundary $(\partial \Omega)^{D}$. For a complete development a discussion on these subjects, including fractional Sobolev spaces, see [37]. Suppose that both $\Omega_{0}^{s}$ and $\Omega^{f}$ are Lipschitz domains.

Next, the standard elasticity bilinear form (cf. (2.3)) is introduced for the elasticity part of the FSI problem:

$$
a_{\Omega_{0}^{s}}(\mathbf{u}, \mathbf{w})=\int_{\Omega_{0}^{s}}(\mathrm{C}: \mathbf{e}(\mathbf{u})): \mathbf{e}(\mathbf{w}) d \mathbf{p} .
$$

For the Stokes part the bilinear form

$$
D_{\Omega^{f}}(\mathbf{v}, \mathbf{w})=\int_{\Omega^{f}} \mu \nabla \mathbf{v}: \nabla \mathbf{w} d \mathbf{x}
$$

is introduced. To write a weak form of the FSI problem, observe that the interface condition (2.19) can be treated as a nonlinear Neumann boundary condition for the solid problem only. Accordingly, we introduce the nonlinear form:

$$
g_{\Gamma_{0}^{I}}(\mathbf{v}, \mathbf{u}, p, \mathbf{w})=\int_{\Gamma_{0}^{I}}\left\{\operatorname{det}(\nabla \mathbf{u}+\mathbf{I})(-p \mathbf{I}+2 \mu \mathbf{e}(\mathbf{v}))(\nabla \mathbf{u}+\mathbf{I})^{-T} \mathbf{n}_{0}\right\} \cdot \mathbf{w} d s .
$$

Finally, let $\mathbf{b}_{0} \in\left[H^{-1}\left(\Omega^{s}\right)\right]^{d}$ be a distributed body force in the solid domain (specified in the reference configuration) and $\mathbf{b} \in\left[H^{-1}\left(\Omega^{f}\right)\right]^{d}$ be a distributed body force in the fluid domain (specified in the deformed configuration). After integrating by parts the balance of linear momentum for the solid and fluid, it is trivial to check that the boundary value problems (2.18)-(2.19) can be restated in the following weak form: Find the interface $\Gamma^{I}$, the deformed configuration of the fluid domain $\Omega^{f}$, the displacements $\mathbf{u} \in\left[H^{1}\left(\Omega_{0}^{s}\right)\right]^{d}$, velocity $\mathbf{v} \in\left[H^{1}\left(\Omega^{f}\right)\right]^{d}$, and pressure $p \in L_{0}^{2}\left(\Omega^{f}\right)$ such that

$$
\begin{aligned}
D_{\Omega^{f}}(\mathbf{v}, \mathbf{w})-(p, \nabla \cdot \mathbf{w})_{\Omega^{f}} & =(\mathbf{b}, \mathbf{w})_{\Omega^{f}} & & \forall \mathbf{w} \in\left[H_{D}^{1}\left(\Omega^{f}\right)\right]^{d} \\
(\nabla \cdot \mathbf{v}, q)_{\Omega^{f}} & =0 & & \forall q \in L_{0}^{2}, \\
a_{\Omega_{0}^{s}}(\mathbf{u}, \mathbf{w}) & =\left(\mathbf{b}_{0}, \mathbf{w}\right)_{\Omega_{0}^{s}}+g_{\Gamma_{0}^{I}}(\mathbf{v}, \mathbf{u}, p, \mathbf{w}) & & \forall \mathbf{w} \in\left[H_{D}^{1}\left(\Omega_{0}^{s}\right)\right]^{d} \\
\Gamma & =\left\{\mathbf{p}+\mathbf{u}(\mathbf{p}) \mid \forall \mathbf{p} \in \Gamma_{0}\right\} . & &
\end{aligned}
$$

In addition $\mathbf{v}, p$, and $\mathbf{u}$ should also satisfy the appropriate boundary conditions specified on $\partial \Omega \backslash \Gamma^{I}$.

5.2. Discretization of the field variables. In this section the finite element method (FEM) approximation for the FSI problem is introduced. Both the solid and the fluid problems are discretized using the FEM method. The elasticity problem is solved by standard linear triangular elements. That is, given a triangulation $\mathcal{T}_{h}^{s}$ of $\Omega_{0}^{s}$, the finite-dimensional approximation space for the displacements is chosen to be

$$
U_{\mathbf{u}}=\left[\left\{u \in C^{0}\left(\Omega_{0}^{s}\right) \mid u \text { is linear on } \forall \tau \in \mathcal{T}_{h}^{s}\right\}\right]^{d} \subset\left[H^{1}\left(\Omega_{0}^{s}\right)\right]^{d} .
$$


The Stokes problem is solved using the $P_{2} P_{1}$ (Taylor-Hood) element pair. Given a triangulation $\mathcal{T}_{h}^{f}$ of $\Omega^{f}$ the approximation spaces for the velocity and pressure are defined by

$$
\begin{aligned}
& U_{\mathbf{v}}=\left[\left\{v \in C^{0}\left(\Omega^{f}\right) \mid v \text { is quadratic polynomial } \forall \tau \in \mathcal{T}_{h}^{f}\right\}\right]^{d} \subset\left[H^{1}\left(\Omega^{f}\right)\right]^{d}, \\
& U_{p}=\left\{p \in C^{0}\left(\Omega^{f}\right) \mid p \text { is linear } \forall \tau \in \mathcal{T}_{h}\right\} \subset H^{1}\left(\Omega^{f}\right) \subset L^{2}\left(\Omega^{f}\right),
\end{aligned}
$$

respectively. This element pair satisfies the so-called inf-sup condition (cf., e.g., [24]); that is, it provides a stable approximation to the Stokes problem alone. While many other elements are known to be LBB stable [27], the Taylor-Hood element was chosen because it provides a balanced approximation for both velocity and pressure [5].

Having defined the finite-dimensional subspaces for the displacements, velocity, and pressure, $U_{\mathbf{u}}, U_{\mathbf{v}}$, and $U_{p}$, respectively, the first three equations in (5.2) lead to the following nonlinear system of algebraic equations:

$$
\left(\begin{array}{ccc}
\mathbf{A}(\mathbf{u}) & \mathbf{C}^{T}(\mathbf{u}) & \mathbf{0} \\
\mathbf{C}(\mathbf{u}) & \mathbf{0} & \mathbf{0} \\
\mathbf{0} & \mathbf{0} & \mathbf{K}
\end{array}\right)\left(\begin{array}{l}
\mathbf{v} \\
\mathbf{p} \\
\mathbf{u}
\end{array}\right)=\left(\begin{array}{c}
\mathbf{b}_{f}(\mathbf{u}) \\
\mathbf{d} \\
\mathbf{b}_{s}+\mathbf{g}(\mathbf{u}, \mathbf{v}, \mathbf{p})
\end{array}\right)
$$

where the blocks $\mathbf{A}(\mathbf{u})$ and $\mathbf{K}$ correspond to the bilinear forms $D_{\Omega^{f}}(\cdot, \cdot)$ and $a_{\Omega_{0}^{s}}(\cdot, \cdot)$, while the blocks $\mathbf{C}(\mathbf{u})$ and $\mathbf{C}(\mathbf{u})^{T}$ couple the velocity and pressure unknowns. Since the position of $\Gamma^{I}$ and hence $\Omega^{f}$ depends on $\mathbf{u}$, both $\mathbf{A}$ and $\mathbf{C}$ are functions of the displacement. The vector columns $\mathbf{b}_{f}(\mathbf{u})$ and $\mathbf{b}_{s}$ correspond to the body force in the fluid and solid, respectively, modified by application of essential (Dirichlet, periodic, etc.) boundary conditions. Note that the vector $\mathbf{d}$ appears in the right-hand side of (5.6) when the essential boundary conditions are applied by matrix transformations. If, for example, they are applied by a penalty method, then $\mathbf{d} \equiv \mathbf{0}$.

It is important to observe that the coupling between the fluid and the structure (2.16) appears on the right-hand side of (5.6) as the nonlinear vector function $\mathbf{g}(\mathbf{u}, \mathbf{v}, \mathbf{p})$ which corresponds to the form $g_{\Gamma_{0}^{I}}(\cdot, \cdot, \cdot, \cdot)$.

5.3. Iterative solution to the FSI problem. One way to solve the boundary value problems $(2.18),(2.17)$ is to use an iterative scheme which successively solves separate problem on the two domains. Consider the following iterative process: Set $\mathbf{u}_{0}=\mathbf{0}, \mathbf{v}_{0}=\mathbf{0}, \mathbf{p}_{0}=0$; given $\left(\mathbf{u}_{k}, \mathbf{v}_{k}, p_{k}\right)^{T}$, find $\left(\mathbf{u}_{k+1}, \mathbf{v}_{k+1}, p_{k+1}\right)^{T}$ such that

$$
\left(\begin{array}{ccc}
\mathbf{A}\left(\mathbf{u}_{k}\right) & \mathbf{C}^{T}\left(\mathbf{u}_{k}\right) & \mathbf{0} \\
\mathbf{C}\left(\mathbf{u}_{k}\right) & \mathbf{0} & \mathbf{0} \\
\mathbf{0} & \mathbf{0} & \mathbf{K}
\end{array}\right)\left(\begin{array}{l}
\mathbf{v}_{k+1} \\
\mathbf{p}_{k+1} \\
\mathbf{u}_{k+1}
\end{array}\right)=\left(\begin{array}{c}
\mathbf{b}_{f}\left(\mathbf{u}_{k}\right) \\
\mathbf{d} \\
\mathbf{b}_{s}+\mathbf{g}\left(\mathbf{u}_{k}, \mathbf{v}_{k+1}, \mathbf{p}_{k+1}\right)
\end{array}\right) .
$$

Continue the iteration until the interface $\Gamma_{k}^{I} \rightarrow \Gamma^{I}$.

Since the matrix on the left-hand side of the above equation is block diagonal, the block corresponding to the fluid is solved first:

$$
\left(\begin{array}{l}
\mathbf{v}_{k+1} \\
\mathbf{p}_{k+1}
\end{array}\right)=\left(\begin{array}{cc}
\mathbf{A}\left(\mathbf{u}_{k}\right) & \mathbf{C}^{T}\left(\mathbf{u}_{k}\right) \\
\mathbf{C}\left(\mathbf{u}_{k}\right) & \mathbf{0}
\end{array}\right)^{-1}\left(\begin{array}{c}
\mathbf{b}_{f}\left(\mathbf{u}_{k}\right) \\
\mathbf{d}
\end{array}\right)
$$

This step corresponds to solving the Stokes equation in the fluid domain, treating the solid as a rigid body. Once $\mathbf{v}_{k+1}$ and $\mathbf{p}_{k+1}$ are available, one can evaluate the

\footnotetext{
${ }^{4}$ The inf-sup condition is also referred to as the Ladyzhenskaya-Babuska-Brezzi (LBB) condition.
} 
nonlinear form $g(\cdots)$. This is equivalent to computing the forces $\mathbf{s}_{k}$ acting on the boundary of the solid part of the domain. Note that by definition of $g(5.1)$, it correctly transfers the fluid stresses $\mathbf{t}_{k}=\mathbf{T}_{k} \mathbf{n}_{k}$ defined by (2.11) in the deformed configuration to their equivalent $\mathbf{s}_{k}=\mathbf{S}_{k} \mathbf{n}_{0}$ in the reference configuration of the solid. Then the block corresponding to the solid, i.e.,

$$
\mathbf{u}_{k+1}=\mathbf{K}^{-1}\left(\mathbf{b}_{s}+\mathbf{g}\left(\mathbf{u}_{k}, \mathbf{v}_{k+1}, \mathbf{p}_{k+1}\right)\right) \text {, }
$$

is solved (in the reference solid configuration), leading to a new iterate $\mathbf{u}_{k+1}$ which satisfies the balance of linear momentum (2.7) in $\Omega_{k}^{s}$ with $\mathbf{S n}_{0}=\mathbf{s}_{k}$. Once $\mathbf{u}_{k+1}$ is available, the new position of the interface can be computed:

$$
\Gamma_{k+1}^{I}=\left\{\mathbf{p}+\mathbf{u}_{k+1}(\mathbf{p}) \mid \forall p \in \Gamma_{0}^{I}\right\} .
$$

This implies that a new fluid domain $\Omega_{k+1}^{f}$ is available and the iterative step (5.7) can be repeated again, until the interface converges to a fixed position. It is clear that if the interface converges, we will have a velocity and a pressure field which satisfy the Stokes equations (2.12), (2.13) and a displacement field which satisfies the elasticity equation (2.7), and, as a result of the converged interface, the interface condition (2.16) will also be satisfied.

The two discretized domains have piecewise straight boundaries, and so the use of linear approximation for the displacement field (5.3) simplifies things because $\Gamma_{k}^{I}$ will remain piecewise straight at each iteration (5.7) and the two meshes will be pointwise conforming at all times. The interface condition (2.14), however, is enforced only weakly, because the stresses in the solid are piecewise constants while the stresses in the fluid are piecewise linear functions. ${ }^{5}$ Note that both are discontinuous across elements. Investigation of curvilinear interface boundaries and/or higher-order approximation spaces for the elasticity problem was outside the scope of this work.

5.3.1. Solution methods for the fluid and solid subproblems. Clearly the above iteration (5.7) requires the solution, at each step, of the linear systems (5.8) and (5.9), corresponding to the Stokes and elasticity subproblems, respectively. In general, the block matrices appearing in, (5.7) are large sparse matrices, and so they can be stored efficiently in machine memory; however, inverting them explicitly is computationally expensive, and the resulting matrices are dense. Therefore, at each iteration (5.7) instead of computing and applying directly the inverse matrices in (5.8) and (5.9) one solves the equivalent linear systems by an iterative method. Since these two systems have to be solved once at each iteration of (5.7), it is important that this is done efficiently.

The solution of linear systems arising in standard FEM discretizations such as linear triangles is a fairly standard subject (cf., e.g., [4]). The method used here for solving (5.9) is the one of $[10,41]$. It uses a preconditioned conjugate gradient (PCG) method $([29,33,25,49])$, with a particular $M I C(0)$ factorization on the diagonal blocks of $\mathbf{K}$. For a detailed description of the $M I C(0)$ preconditioner the reader is referred to [10]. The Stokes system (5.8) is more complicated to solve efficiently $[14,53,5]$. The method used in this work is to solve it with a PCG iteration for the pressure Schur complement $[53,39]$. The preconditioner is a mass matrix on the pressure space [53]. Each PCG iteration requires an inversion of the velocity matrix A, which is done by a second, internal PCG, preconditioned by an ILU preconditioner [49]. A detailed description of the entire solution procedure can be found in [48].

\footnotetext{
${ }^{5}$ Since the contributions to the stresses in the fluid come both from the pressure and the velocity gradients, things will not change if one uses other approximations for the fluid, e.g., $P_{2} P_{0}$.
} 


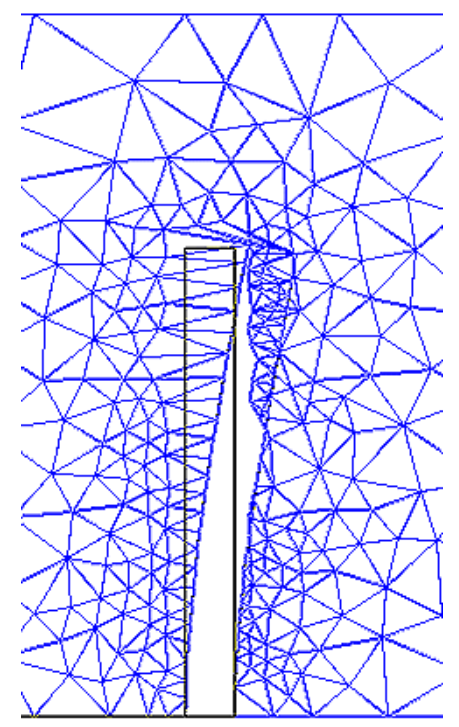

(a) Degenerated mesh

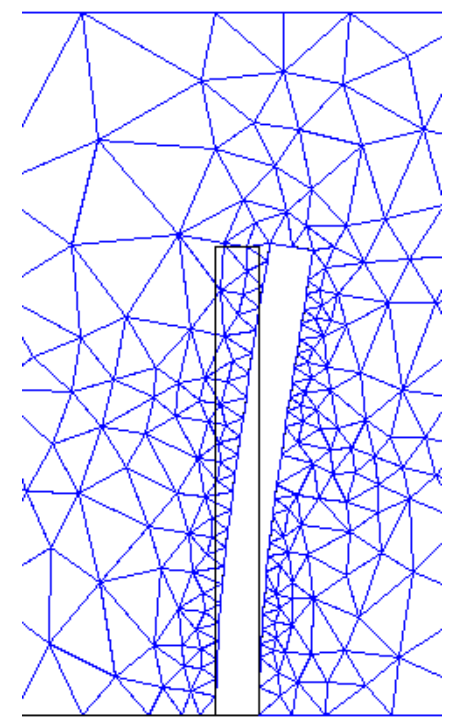

(b) Regenerated mesh

FIG. 5. A two-dimensional degenerate mesh. In this example an elastic obstacle deforms to the left in response to flow in the channel. The solid lines indicate its initial configuration. If only the boundary nodes of the fluid mesh are moved, it degenerates (left). The second mesh (right) is obtained after remeshing the fluid domain.

5.3.2. Fluid mesh regeneration. At the beginning of the iteration (5.7) one has a conforming triangulation of both the solid and the fluid domains. At the end of each iterative step the fluid domain is updated, and as a result, the mesh also has to change. On the other hand, the elasticity equation is always solved in the original configuration, and so the elastic mesh remains unchanged. Since only conforming meshes are considered, the modification of the fluid mesh must be such that conformity is maintained on $\Gamma^{I}$. That is, when the interface is deformed using the computed displacement $\mathbf{u}_{k+1}$, solid vertices will coincide with fluid vertices, and solid segments (faces in three dimensions) should coincide with fluid ones.

The easiest approach is to move the interface vertices of the fluid mesh, which will affect only the elements which contain them. This will work as long as the interface displacements are small compared to the local mesh size. If the mesh size near the interface is comparable to the displacements of the interface, the mesh can lose quality or completely degenerate if a vertex is moved into another element (Figures 5 and 6). As a result, given the domain and boundary condition, the mesh size in the fluid domain cannot be too small. Such a restriction is clearly unacceptable. It can be overcome either by globally modifying the existing mesh, for example, by solving an artificial elasticity problem in the fluid domain, or by remeshing it (locally or globally).

The global remeshing approach is selected here because of the ready availability of mesh generators which could do that. As the new fluid domain $\Omega_{k+1}^{f}$ is computed at the end of each step (5.7), the elements with vertices on the interface are modified, and if the resulting mesh has poor quality and/or it degenerates, then the entire fluid domain is remeshed, retaining the same boundary segments in two dimensions and 


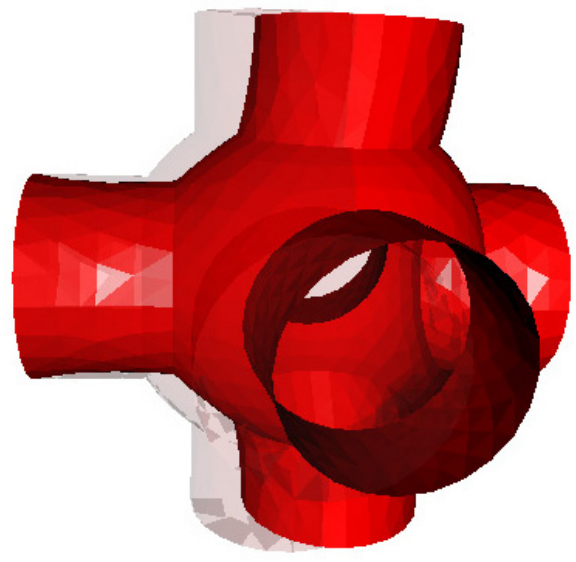

(a) Flow external to an elastic skeleton

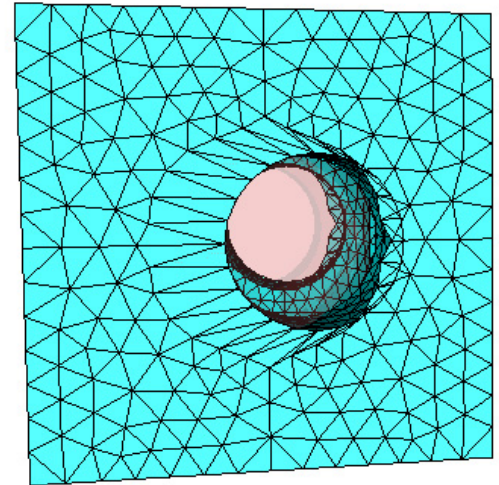

(b) Degenerated mesh after the first iteration

FIG. 6. A three-dimensional degenerate mesh. Another case when the mesh degenerates after a new position of the interface is computed. Shown are the initial (transparent shade) and final position (darke $\bar{\equiv}$ de) of the interface $\Gamma^{I}$ (a) and the degenerated mesh after the first iteration (b). The domatw the unit cube, and the solid geometry is formed by the intersection of three perpendicular cylinders and a central circle. The flow is from left to right and is exterior to the solid (the caps on the cylinders are not shown), and the boundary conditions allow the caps of the two cylinders perpendicular to the flow direction to slide on the side of the unit cube. Unlike the two-dimensional case, the regeneration of the mesh involves both the interior and part of the surface mesh.

the same interface faces in three dimensions. Note that in three dimensions, in addition to the entire volume mesh, one also has to modify part of the surface mesh, as can be seen from Figure 6, while keeping the interface conforming. In principle local regeneration of the mesh (after removing low quality/degenerate elements) is an interesting possibility which can significantly speed up the process; however, developing the software necessary to utilize this strategy was outside the scope of this work.

6. Numerical examples. Several numerical tests related to upscaling of flow in deformable porous media are discussed here. It is shown that the developed solver can be used for solving cell problems related to the poroelasticity as well as for solving microscale problems related to the above derived nonlinear Darcy law.

Three model problems are considered in order to test the iterative scheme of section 5.3. The first two model problems involve flow in the elastic channel geometry of section 4, while the third one is for a channel with an elastic segment. The first model problem is used to demonstrate the convergence properties of the iterative scheme with regard to various problem parameters. The second problem is set up in the same type of geometry, but with slightly different boundary conditions, so that a comparison can be made between the numerical solutions and the asymptotic solution of section 4 . The last problem of flow in a channel with an elastic segment demonstrates the highly nonlinear dependence of permeability on the pressure gradient across the channel. 


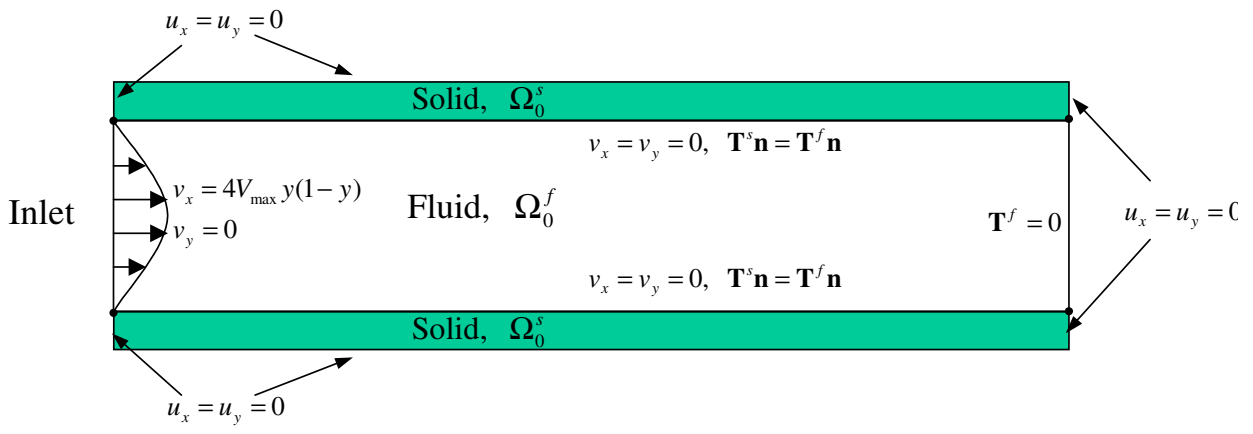

FIG. 7. Model problem geometry.

6.1. Flow through an elastic channel. The first model problem to be considered is one for a flow through an elastic channel. The geometry is shown on Figure 7. The channel has length 5 and thickness 1 . The thickness of each of the elastic slabs is 0.2 . The material parameters used for the solid are $E=1.44$ and $\nu=0.2$, while the fluid has viscosity $\mu=0.1$ and density $\rho=1$. The velocity distribution at the inlet is that of a developed Poiseuille flow: $v_{x}=4 * V_{\max } y(1-y)$, where the constant $V_{\max }$ is the maximal inflow velocity (achieved at $y=0.5$ ).

First, the numerical algorithm is tested for $V_{\max }=1 / 4$ and a (triangular) mesh with $h \sim 16 * 10^{-2}$ in the fluid domain and $h \sim 4 * 10^{-2}$ in the solid domain. No triangle had internal angles less than 30 degrees. The mesh is chosen coarser in the fluid domain because of the higher-order accuracy of the fluid discretization in comparison with the piecewise linear approximation for the solid problem. ${ }^{6}$ The computed pressure and velocity profiles are displayed in Figure 8(a)-8(b).

Next, the problem is solved for several values of $V_{\max }$ and three different mesh sizes. This is done in order to get a preliminary idea of the convergence rate sensitivity of the iterative scheme to the inflow velocity and mesh size. The number of iterations it took to reach relative precision $10^{-6}$ is reported in Table 6.1. Also given in the table is the maximum vertical displacement in the solid. Based on the nondimensional analysis of section 4 for this problem, varying input velocity $V_{\max }$ is the same as varying the elastic stiffness $E$ or fluid viscosity $\mu$.

It can be concluded from this table that the iteration numbers depend on the inlet velocity which in turn directly affects the magnitude of the interface displacement. In general, numerical experiments suggest that the important parameter is the magnitude of the interface displacements. On the other hand, the iteration convergence rate does not seem to depend on the mesh parameters. The iteration numbers for the remaining numerical examples in sections 6.2 and 6.3 followed a similar pattern and are not reported.

Finally, the authors are not aware of any general results on the convergence of the iterative algorithm of section 5.3. However, using, for example, the maximum displacement of the interface one sees that it stabilizes as $h$ decreases for each case of inlet velocity, which can be interpreted as an indication that the algorithm is convergent.

\footnotetext{
${ }^{6}$ It is difficult to access the overall accuracy of the complete FSI scheme. The Taylor-Hood approximation of the fluid problem alone, given sufficiently smooth data, is third-order accurate for the velocity and second-order accurate for the pressure [54]. However, the piecewise linear discretization of the interface should decrease the overall order of the scheme.
} 


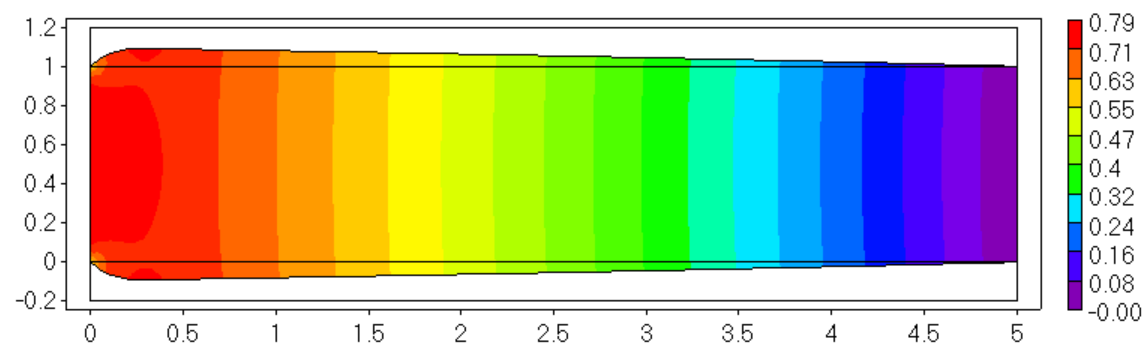

(a) Pressure profile

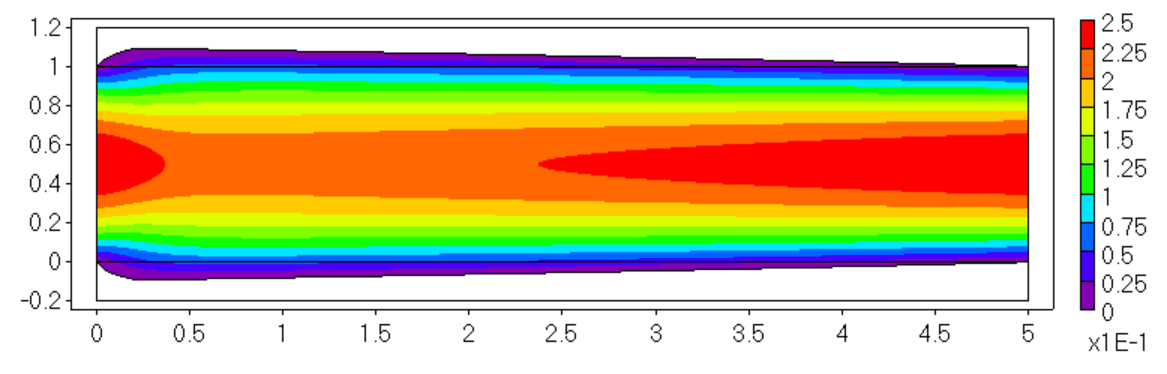

(b) Horizontal velocity profile

FIG. 8. Solution of the FSI problem for $V_{\max }=1 / 4$. Shown are the velocity and pressure in the deformed configuration of the fluid domain $\Omega^{f}$.

TABle 6.1

\begin{tabular}{|l|c|c|c|c|c|c|}
\hline \multirow{2}{*}{$V_{\max }$} & \multicolumn{2}{|c|}{$h$} & \multicolumn{2}{c|}{$h / 2$} & \multicolumn{2}{c|}{$h / 4$} \\
\cline { 2 - 7 } & Iterations & $\max u_{y}$ & Iterations & $\max u_{y}$ & Iterations & $\max u_{y}$ \\
\hline $1 / 4$ & 9 & 0.0894687 & 9 & 0.0894628 & 9 & 0.0894809 \\
\hline $1 / 8$ & 7 & 0.0504095 & 7 & 0.0505136 & 7 & 0.0505101 \\
\hline $1 / 16$ & 6 & 0.0271444 & 6 & 0.0271742 & 6 & 0.0271846 \\
\hline $1 / 32$ & 5 & 0.0141473 & 5 & 0.0141589 & 5 & 0.0141654 \\
\hline $1 / 64$ & 5 & 0.0072323 & 5 & 0.0072312 & 5 & 0.0072402 \\
\hline
\end{tabular}

The next example verifies this much more rigorously by using the asymptotic solution developed in section 4 .

6.2. Permeability of porous medium formed by parallel arrangement of long elastic channels. Recall that one of the first derivations of Darcy's law for flow in rigid porous media was done for a medium which is an arrangement of long parallel channels with rigid walls: Darcy's law follows by averaging Poiseuille flow in these channels. In a similar way, we will evaluate the pressure drop/flow rate dependence for a single long channel with elastic walls; permeability of a porous medium formed by such channels can be obtained straightforwardly. 
While the figures presented in the previous numerical example look reasonable from a physical standpoint, in the absence of exact analytical solutions it is difficult to verify the quality of the numerical solution. It may happen that the continuous problem (5.2) is not well posed. It may also happen that the selected numerical approximations (5.3)-(5.5) are not stable ones; that is, the stationary FSI problem (5.2) does have a unique solution, but the FEM subspaces (5.3)-(5.5) do not lead to a convergent method.

The asymptotic solution developed in section 4 for a long elastic channel can, however, be compared with a numerical one. The asymptotic formulae (4.51) and (4.56) are derived based on several assumptions (see section 4) and without analysis of the rate of convergence with respect to the small parameter $\varepsilon$. However, if both the asymptotic solution and the numerical one converge to the actual one, then we should also observe convergence between the two of them. In this section we use the numerical method described in section 5.3 in order to validate the asymptotic formulae (4.51) and (4.56) and vice versa.

To do this, the elastic channel of Figure 4 is discretized for $l=\delta=0.5$. In this way, a $y$-periodic arrangement of this geometry will have solid and fluid regions of equal unit width. The boundary conditions are also modified, compared to the previous model problem. Instead of fixing both displacements at $x=0$ and $x=L$ we constrain only $u_{1}$; i.e., the end of the channel is now free to move in the vertical direction. Note that this does not represent a $y$-periodic boundary value problem, because $u_{1}=0$ at $y= \pm(l+\delta)$. Also, the boundary condition for the fluid at the inlet $x=0$ is a prescribed pressure, i.e, $p(0, y)=P^{0}$. As in section 4 the half-width of the channel in the deformed configuration is denoted by $\gamma(x)$.

The asymptotic expansion depends on two parameters: $P^{0}$ and $\varepsilon=l / L$. Several numerical results comparing the computed values for $\gamma_{h}, K_{h}$ with the analytical ones $\gamma, K$ are given in Tables 6.2 and 6.3. The first shows the $L^{2}$ norm of the error and

TABLE 6.2

$L^{2}$ error along the interface between the numerical and asymptotic results of a long elastic channel.

\begin{tabular}{|c|c|c|c|c|}
\hline \multirow{2}{*}{$P^{0}$} & \multicolumn{2}{|c|}{$\varepsilon=\frac{1}{10}(l=0.5, L=5)$} & \multicolumn{2}{c|}{$\varepsilon=\frac{1}{20}(l=0.5, L=10)$} \\
\cline { 2 - 5 } & $\frac{\left\|\gamma_{h}-\gamma\right\|_{L^{2}}}{\|\gamma\|_{L^{2}}}$ & $\frac{\left|K_{h}-K\right|}{K}$ & $\frac{\left\|\gamma_{h}-\gamma\right\|_{L^{2}}}{\|\gamma\|_{L^{2}}}$ & $\frac{\left|K_{h}-K\right|}{K}$ \\
\hline 0.32 & $2.41 \times 10^{-3}$ & $6.63 \times 10^{-3}$ & $8.47 \times 10^{-4}$ & $1.82 \times 10^{-3}$ \\
\hline 0.16 & $1.19 \times 10^{-3}$ & $3.33 \times 10^{-3}$ & $4.21 \times 10^{-4}$ & $1.06 \times 10^{-3}$ \\
\hline 0.08 & $5.96 \times 10^{-4}$ & $1.65 \times 10^{-3}$ & $2.10 \times 10^{-4}$ & $5.34 \times 10^{-4}$ \\
\hline 0.04 & $2.98 \times 10^{-4}$ & $8.19 \times 10^{-4}$ & $1.05 \times 10^{-4}$ & $2.68 \times 10^{-4}$ \\
\hline
\end{tabular}

TABLE 6.3

Pointwise comparisons of numerical and asymptotic results of a long elastic channel.

\begin{tabular}{|c|c|c|c|c|}
\hline \multirow{2}{*}{$P^{0}$} & \multicolumn{2}{|c|}{$\varepsilon=\frac{1}{10}(l=0.5, L=5)$} & \multicolumn{2}{c|}{$\varepsilon=\frac{1}{20}(l=0.5, L=10)$} \\
\cline { 2 - 5 } & $\frac{\left|\gamma_{h}(1)-\gamma(1)\right|}{|\gamma(1)|}$ & $\frac{\left|K_{h}(1)-K(1)\right|}{|K(1)|}$ & $\frac{\left|\gamma_{h}(2)-\gamma(2)\right|}{|\gamma(2)|}$ & $\frac{\left|K_{h}(2)-K(2)\right|}{|K(2)|}$ \\
\hline 0.32 & $2.59 \times 10^{-4}$ & $6.02 \times 10^{-4}$ & $8.97 \times 10^{-5}$ & $2.57 \times 10^{-4}$ \\
\hline 0.16 & $4.58 \times 10^{-5}$ & $1.71 \times 10^{-5}$ & $2.49 \times 10^{-5}$ & $8.15 \times 10^{-5}$ \\
\hline 0.08 & $5.02 \times 10^{-6}$ & $5.79 \times 10^{-5}$ & $5.85 \times 10^{-6}$ & $6.56 \times 10^{-6}$ \\
\hline 0.04 & $1.06 \times 10^{-5}$ & $4.38 \times 10^{-5}$ & $4.76 \times 10^{-7}$ & $1.00 \times 10^{-6}$ \\
\hline
\end{tabular}


the second one the error at a fixed point $x=0.2 * L$. The numerical solutions used were consequently refined, until the discretization error did not influence the first two digits of the results. It can be seen from Table 6.2 that formulae (4.51) and (4.56) are in very good agreement with the numerically computed solution to the FSI problem. This indicates that as $\varepsilon \rightarrow 0$ and $h \rightarrow 0$ both the asymptotic and the numerical solution to the FSI problem converge to the actual one.

6.3. Flow through a channel with an elastic segment. In this problem a channel with a deformable segment (Figure 9) is considered. The channel has height $H=1$ and length $L=14$, and an elastic segment is located in the middle of the top channel wall. The segment has length 10, thickness 0.1 , and elastic properties $E=12 * 10^{6}$ and $\nu=0.2$. The fluid viscosity and density are again $\mu=\rho=1$. The segment is built into the rest of the wall at its two ends. The appropriate boundary conditions at the ends of the segment are therefore homogeneous Dirichlet boundary conditions for both displacements. Natural boundary conditions (zero tractions) are imposed on the top side of the segment. The lower side is the fluid-structure interface $\Gamma_{0}^{I}$. The input flow velocity is again a developed Poiseuille flow: $v_{x}=\frac{6}{14} Q y(1-y)$, with

$$
Q=\int_{\Omega} v_{x} d \mathbf{x}
$$

being the total volumetric flow rate. The right end of the channel has an outflow boundary condition $\left(\mathbf{T}^{f}=\mathbf{0}\right)$. The purpose of this problem is to investigate the permeability of the channel at various values for $Q$. In the case of an entirely rigid channel the pressure drop $\Delta p / L$ is proportional to the mass flux $Q$. Since the coupled problem is nonlinear it is expected that for sufficiently large deformations of the interface the resulting mass flow for a given pressure drop will depart from the linear relationship of the Darcy law. The FSI problem is solved for several different values of $Q$, and the resulting pressure, pressure gradients in the fluid, and displacements in the solid are computed. Two measures for permeability are considered. One is the ratio of the average velocity and average pressure gradient:

$$
\bar{K}=\frac{Q}{\int_{\Omega} \frac{\partial p}{\partial x} d \mathbf{x}},
$$

which is similar to the standard homogenization results for flow through a rigid skeleton. Since the geometry allows only nonzero net flow in the $x$-direction, it also makes

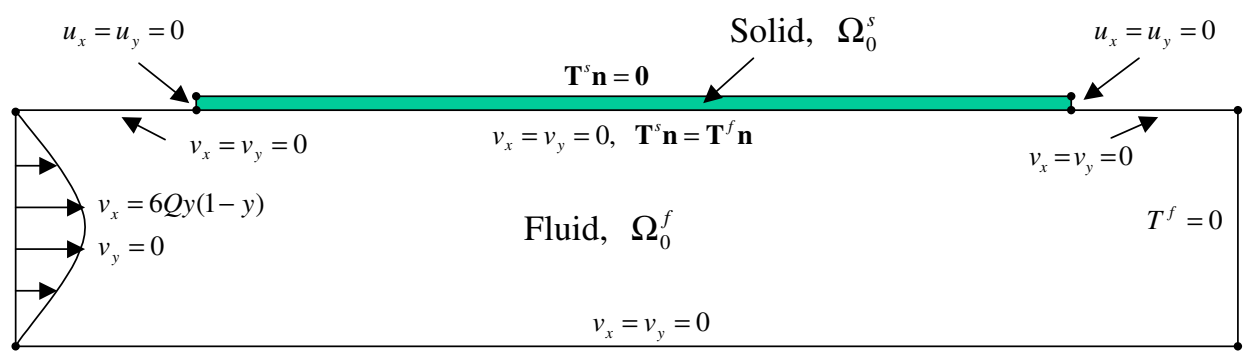

FIG. 9. Geometry of a channel with an elastic segment (figure not drawn to scale). 
sense to consider the pressure drop as an alternative to the average pressure gradient:

$$
\hat{K}=\frac{Q}{\Delta p / L}
$$

While the pressure gradient can be readily calculated from the flow solution, it is not immediately clear how to evaluate the pressure drop across the channel. However, thanks to the selection of material parameters and geometry dimensions the inlet is sufficiently away from the segment, and so the change in the flow downstream hardly affects the inlet pressure distribution, which is essentially constant along the $y$-direction. Similarly, the outlet is sufficiently separated from the end of the elastic segment, and the flow has time to redevelop to the parabolic Poiseuille distribution, as can be seen in Figure 10(a)-(b). The results are summarized on Table 6.4, and it is clear that both permeability measures behave nonlinearly as $Q$ is varied. This can also be seen in Figure 11, which shows $\bar{K}$ and $\hat{K}$ as functions of $Q$. As $Q \rightarrow 0$ both of them tend to the permeability of a straight, rigid channel which can be calculated directly from the Poiseuille solution and in this case is equal to $1 / 12$.

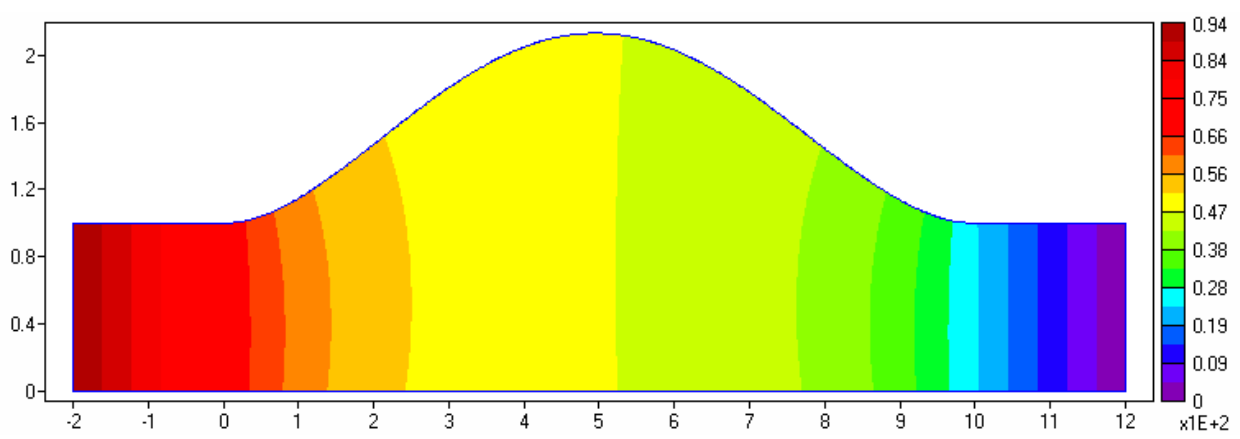

(a) Pressure profile (figure not drawn to scale)

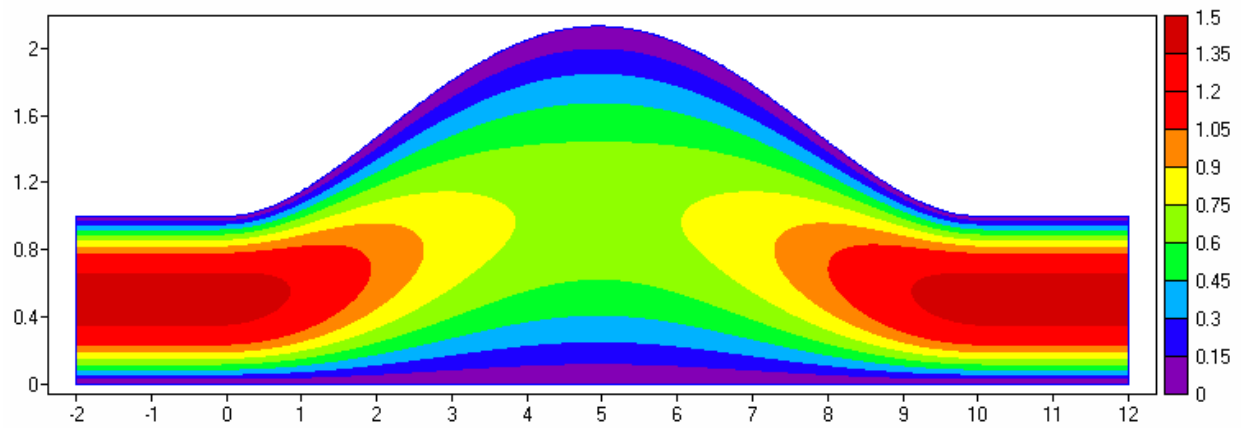

(b) Profile of the horizontal velocity component (figure not drawn to scale)

FIG. 10. Final configuration of the fluid domain $\Omega^{f}$ for $Q=15$. 
TABLE 6.4

\begin{tabular}{|c|c|c|c|c|c|}
\hline$Q$ & $\int_{\Omega} \frac{\partial p}{\partial x} d \mathbf{x}$ & $\left.p\right|_{\text {inlet }}$ & $Q / \int_{\Omega} \frac{\partial p}{\partial x} d \mathbf{x}$ & $\frac{Q}{\Delta p / L}$ & $\max u_{y}$ \\
\hline 14.0000 & -106.0853266 & -93.8605 & 0.13196 & 0.14916 & 1.161 \\
7.0000 & -60.3754287 & -53.9478 & 0.11594 & 0.12975 & 0.651 \\
3.5000 & -33.6888175 & -30.8411 & 0.10389 & 0.11348 & 0.373 \\
1.7500 & -18.3178061 & -17.2482 & 0.09553 & 0.10146 & 0.209 \\
0.8750 & -9.7023494 & -9.35044 & 0.09018 & 0.09358 & 0.113 \\
0.4375 & -5.0280238 & -4.923492 & 0.08701 & 0.08889 & \\
\hline
\end{tabular}

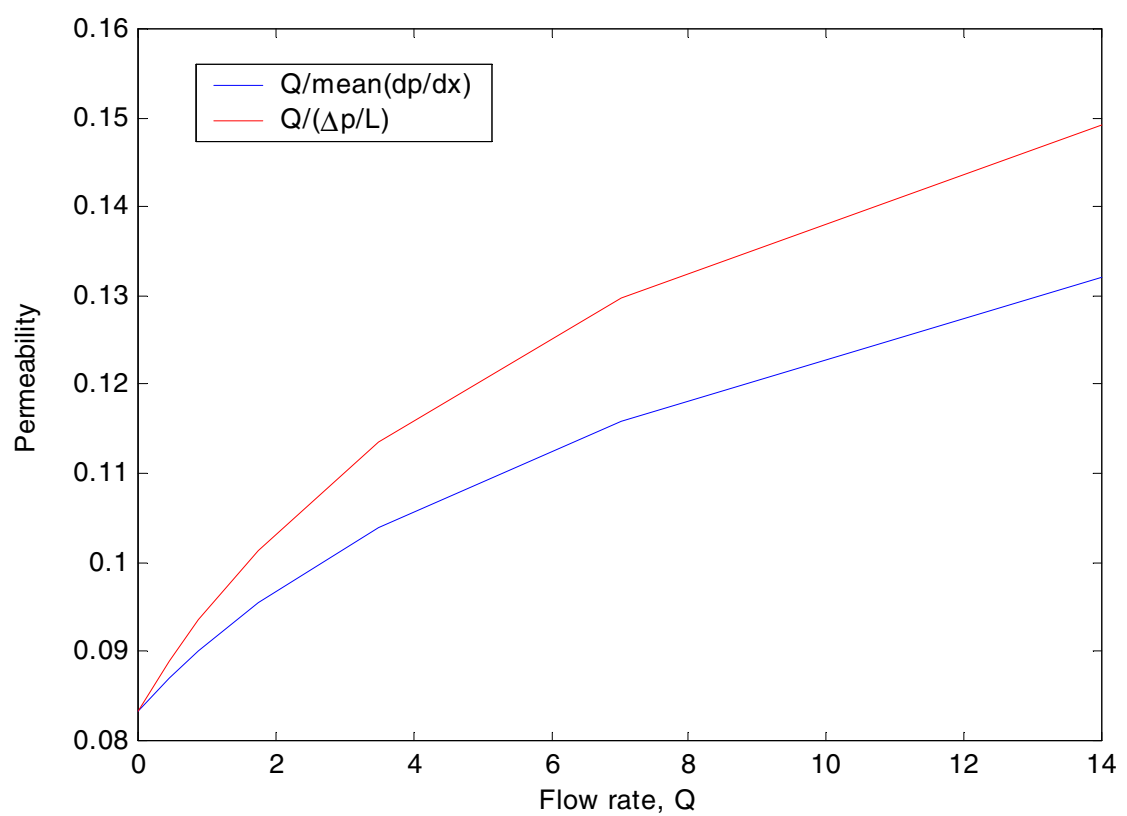

FIG. 11. Permeability of a channel with an elastic segment.

7. Conclusions. In this paper, a poroelastic medium is considered, and the FSI problem at the microscale is presented. The displacements of the fluid-structure interface at the pore level are assumed large enough so that standard homogenization techniques leading to Biot-type equations on the macroscopic scale cannot be applied. An asymptotic analysis is then performed on a long elastic channel, and a nonlinear Darcy-type law for the $y$-averaged pressure is obtained. From this equation a nonlinear upscaled permeability-like functional is identified. This permeability is found to depend on the initial channel geometry, Lamé's constants for the solid, and the $y$-averaged pressure in the channel.

A FEM-based FSI solver is also presented. The solver successfully utilized a Dirichlet-Neumann iterative scheme for solving the FSI problem. Numerical computations are performed which are used to verify the numerical solutions in comparison with an asymptotic solution to the FSI problem. This increases the degree of confidence with which such numerical methods can be used. It also corroborates the asymptotic derivation. 
Work on upscaling the poroelasticity problem and the related FSI equations can progress in multiple directions. When no restrictions are placed on the pore-level displacements, it is unlikely that closed-form macroscopic equations can be obtained for general geometries. However, numerical upscaling can be attempted, and the analysis in this work suggests that the average pressure at the pore level should be the most important upscaling parameter. Further, it will be helpful to compare microscale solutions to the FSI problem with macroscopic solutions of various macroscopic poroelastic equation and determine when the standard homogenization fails.

The iterative algorithm for the FSI problem can also be improved. At the end of each solid sol $\equiv$, one can attempt to perform a line search along the direction of the proposed ikterface displacement and try to correct it so that a certain residual is always reduced. One can also attempt to keep track of several possible interface correction displacements and favor new interface positions which are orthogonal to past displacements. Such work can improve both the convergence rate and the stability of the algorithm.

Acknowledgment. Peter Popov would like to express deep gratitude to Dr. Heiko Andrä for the fruitful discussions during this project.

\section{REFERENCES}

[1] T. Arbogast, Analysis of a two-scale, locally conservative subgrid upscaling for elliptic problems, SIAM J. Numer. Anal., 42 (2004), pp. 576-598.

[2] J. Auriault, P. Royer, and C. Geindreau, Filtration law for power-law fluids in anisotropic porous media, Int. J. Eng. Sci., 40 (2002), pp. 1151-1163.

[3] J.-L. Auriault and E. Sanchez-Palencia, Étude du comportement macroscopique d'un milieu poreux saturé déformable, J. Mécanique, 16 (1977), pp. 575-603.

[4] O. Axelsson And V. A. Barker, Finite Element Solution of Boundary Value Problems: Theory and Computations, Academic Press, London, 1984; reprinted, SIAM, Philadephia, 2001.

[5] O. Axelsson And M. Neytcheva, Preconditioning methods for linear systems arising in constrained optimiziation problems, Numer. Linear Algebra Appl., 10 (2003), pp. 3-31.

[6] N. Bakhvalov and G. Panasenko, Homogenization: Averaging Processes in Periodic Media, Kluwer Academic Publishers, Dordrecht, The Netherlands, 1990.

[7] J. Bear, Dynamics of Fluids in Porous Media, Dover, New York, 1972.

[8] A. Bensoussan, J. Lions, And G. Papanicolaou, Asymptotic Analysis for Periodic Structures, Stud. Math. Appl. 5, North-Holland, Amsterdam, 1978.

[9] M. Biот, General theory of three dimensional consolidation, J. Appl. Phys., 12 (1941), pp. 155-164.

[10] R. Blaheta, Displacement decompostion - incomplete factorization preconditioning techniques for linear elasticity problems, Numer. Linear Algebra Appl., 1 (1994), pp. 107-128.

[11] A. Bourgeat, E. Marušić Paloka, And A. Mikelić, Weak nonlinear corrections for Darcy's law, Math. Models Methods Appl. Sci., 6 (1996), pp. 1143-1155.

[12] A. Bourgeat And A. Mikelić, A note on homogenization of Bingham flow through a porous medium, J. Math. Pures Appl., 72 (1993), pp. 405-414.

[13] A. Bourgeat And A. Mikelić, Homogenization of a polymer flow through a porous medium, Nonlinear Anal., 26 (1996), pp. 1221-1253.

[14] J. Bramble and J. Pasciak, Iterative techniques for time dependent stokes problems, Comput. Math. Appl., 33 (1997), pp. 13-30.

[15] R. Burridge And J. Keller, Poroelasticity equations derived from microstructure, J. Acoust. Soc. Amer., 70 (1981), pp. 1140-1146.

[16] T. Clopeau, J. L. Ferrín, R. P. Gilbert, And A. Mikelić, Homogenizing the acoustic properties of the seabed. II, Math. Comput. Modelling 33 (2001), pp. 821-841.

[17] H. DARCy, Les fontaines publique de la ville de Dijon, Librairie des Corps Impériaux des Ponts et Chaussées et des Mines, Paris, 1856.

[18] Y. Efendiev And A. PANKov, Numerical homogenization of monotone elliptic operators, Multiscale Model. Simul., 2 (2003), pp. 62-79. 
[19] H. EnE, Application of the homogenization method to transport in porous media, in Dynamics of Fluids in Hierarchical Porous Media, J. Cushman, ed., Academic Press, London, 1990, pp. 223-241.

[20] J. L. Ferrín AND A. Mikelić, Homogenizing the acoustic properties of a porous matrix containing an incompressible inviscid fluid, Math. Methods Appl. Sci., 26 (2003), pp. 831-859.

[21] M. Firdaouss And J. Guermond, Homogenization of the Navier-Stokes equations at low Reynolds number, C. R. Acad. Sci. Paris Sér. I Math., 320 (1995), pp. 245-251.

[22] P. Forchneimer, Wasserbewegung durch boden, Z. Ver. Deutsch. Ing., 45 (1901), pp. $1782-1788$.

[23] R. P. Gilbert And A. Mikelić, Homogenizing the acoustic properties of the seabed. I, Nonlinear Anal. 40 (2000), pp. 185-212.

[24] V. Girault and P. Raviart, Finite Element Methods for Navier-Stokes Equations, SpringerVerlag, Berlin, 1986.

[25] G. H. Golub And C. F. Van Loan, Matrix Computations, The Johns Hopkins University Press, Baltimore, MD, 1996.

[26] C. Grandmont, Existence for a three-dimensional steady state fluid-structure interaction problem, J. Math. Fluid Mech., 4 (2002), pp. 76-94.

[27] P. M. Gresho And R. L. SAni, Incompressible Flow and the Finite Element Method: Advection-Diffusion and Isothermal Laminar Flow, John Wiley and Sons, Chichester, UK, 1998.

[28] M. E. Gurtin, An Introduction to Continuum Mechanics, Academic Press, San Diego, CA, 1981.

[29] M. Hesteness And E. Stiefel, Methods of conjugate gradients for solving linear systems, J. Research Nat. Bur. Standards, 49 (1952), pp. 409-436.

[30] U. Hornung, Homogenization and Porous Media, Interdiscip. Appl. Math. 6, Springer-Verlag, New York, 1996.

[31] O. Iliev And D. Stoyanov, On a flexible tool for upscaling porous media flow problems, J. Theoret. Appl. Mech., 31 (2001), pp. 18-30.

[32] W. JäGer, A. Mikelić, AND N. Neuss, Asymptotic analysis of the laminar viscous flow over a porous bed, SIAM J. Sci. Comput., 22 (2001), pp. 2006-2028.

[33] C. Lanczos, Solution of systems of linear equations by minimized iterations, J. Res. Nat. Bur. Standards, 49 (1952), pp. 33-53.

[34] V. Laptev, Numerical Solution of Coupled Flow in Plain and Porous Media, Ph.D. thesis, Technical University of Kaiserslautern, Kaiserslautern, Germany, 2003.

[35] C. K. Lee And C. C. MeI, Re-examination of the equations of poroelasticity, Int. J. Eng. Sci., 35 (1997), pp. 329-352.

[36] R. Lewis And B. Schrefler, The Finite Element Method in the Static and Dynamic Deformation and Consolidation of Porous Media, John Wiley and Sons, Chichester, UK, 1998.

[37] J. Lions And Magenes, Problèmes aux Limites non Homogènes et Applications, Vol. 1, Dunod, Paris, 1968.

[38] J. Lions And E. Sanchez-Palencia, Flow of a visco-plastic bingham solution in a porous medium, J. Math. Pures Appl. (9), 60 (1981), pp. 341-360.

[39] D. Loghin And A. Wathen, Schur complement preconditioners for the Navier-Stokes equations, Internat. J. Numer. Methods Fluids, 40 (2002), pp. 403-412.

[40] L. E. Malvern, Introduction to the Mechanics of a Continuous Medium, Prentice-Hall, Englewood Cliffs, NJ, 1969.

[41] S. Margenov AND P. Popov, Mic(0) dd preconditioning of fem elasticity problems on nonstructured meshes, in Proceedings of ALGORITHMY 2000, 15th Conference on Scientific Computing, Vysoke Tatry-Podbanske, Slovakia, 2000, pp. 245-253.

[42] C. Mei And J. Auriault, The effect of weak inertia on flow through a porous medium, J. Fluid Mech., 222 (1991), pp. 647-663.

[43] C. Mei And J.-L. Auriault, Mechanics of heterogeneous porous media with several spatial scales, Proc. Roy. Soc. London Ser. A, 426 (1989), pp. 391-423.

[44] A. Mikelić, Homogenization theory and applications to filtration through porous media, in Filtration in Porous Media and Industrial Application, Lecture Notes in Math. 1734, A. Fasano, ed., Springer-Verlag, Berlin, 2000, pp. 127-214.

[45] A. Mikelić, Recent developments in multiscale problems coming from fluid mechanics, in Trends in Nonlinear Analysis, Springer-Verlag, Berlin, 2003, pp. 225-267.

[46] N. Neuss, Multiscale preconditioning, in Mini-Workshop: Numerical Upscaling: Theory and Applications, Mathematisches Forschungsinstitut Oberwolfach, Oberwolfach, Germany, 2005, pp. 1152-1155. 
[47] N. Neuss, W. Jaeger, And G. Wittum, Homogenization and multigrid, Computing, 66 (2002), pp. $1-26$.

[48] P. Popov, Constitutive Modelling of Shape Memory Alloys and Upscaling of Deformable Porous Media, Ph.D. thesis, Texas A\&M University, College Station, TX, 2005.

[49] Y. SAAD, Iterative Methods for Sparse Linear Systems, PWS, New York, 1996.

[50] E. Sanchez-Palencia, Nonhomogeneous Media and Vibration Theory, Lecture Notes in Phys., 127, Springer-Verlag, Berlin, 1980.

[51] E. Sanchez-Palencia And H. Ene, Equations et phénomènes de surface pour l'écoulement dans un modèle de milieu poreux, J. Mécanique, 14 (1975), pp. 73-108.

[52] J. Slattery, Flow of viscoelastic fluids through porous media, AIChE J., 13 (1967), pp. 1066-1071.

[53] S. TuREK, Efficient Solvers for Incompressible Flow Problems: An Algorithmic and Computational Approach, Springer Verlag, New York, 1999.

[54] R. VerfürTh, Error estimates for a mixed finite element approximation of the Stokes equations, RAIRO Anal. Numér., 18 (1984), pp. 175-182.

[55] X. Wu, Y. EFendiev, And L. Durlofsky, Analysis of upscaling absolute permeability, Discrete Contin. Dyn. Syst. Ser. B, 2 (2002), pp. 185-204.

[56] V. Zhikov, S. Kozlov, AND O. Oleinik, Homogenization of Differential Operators and Integral Functionals, Springer-Verlag, Berlin, 1994. 